\title{
Two-dimensional Stokes and Hele-Shaw flows with free surfaces
}

\author{
L. J. CUMMINGS ${ }^{1}$, S. D. HOWISON ${ }^{1}$, and J. R. KING ${ }^{2}$ \\ 1 Mathematical Institute, 24-29 St Giles', Oxford OX1 3LB, UK \\ ${ }^{2}$ Department of Theoretical Mechanics, University of Nottingham, Nottingham NG7 2RD, UK
}

(Received 10 April 2006)

\begin{abstract}
We discuss the application of complex variable methods to Hele-Shaw flows and twodimensional Stokes flows, both with free boundaries. We outline the theory for the former, in the case where surface tension effects at the moving boundary are ignored. We review the application of complex variable methods to Stokes flows both with and without surface tension, and we explore the parallels between the two problems. We give a detailed discussion of conserved quantities for Stokes flows, and relate them to the Schwarz function of the moving boundary and to the Baiocchi transform of the Airy stress function. We compare the results with the corresponding results for Hele-Shaw flows, the principal consequence being that for Hele-Shaw flows the singularities of the Schwarz function are controlled in the physical plane, while for Stokes flow they are controlled in an auxiliary mapping plane. We illustrate the results with the explicit solutions to specific initial value problems. The results shed light on the construction of solutions to Stokes flows with more than one driving singularity, and on the closely related issue of momentum conservation, which is important in Stokes flows, although it does not arise in Hele-Shaw flows. We also discuss blow-up of zero-surface-tension Stokes flows, and consider a class of weak solutions, valid beyond blow-up, which are obtained as the zero-surface-tension limit of flows with positive surface tension.
\end{abstract}

\section{Introduction}

Useful exact solutions to free boundary problems are rare. We discuss two problems for which they are not: two-dimensional Hele-Shaw flows without surface tension, and twodimensional Stokes flow both with and without surface tension. In both cases, ingenious complex variable methods have been developed which use conformal mapping to reformulate the free boundary problem (necessarily posed in an unknown domain) as a nonlinear boundary value problem in a canonical fixed domain such as the unit disc. Remarkably, large classes of exact solutions can be found for both problems; they are generated by polynomials and rational conformal mappings. One theme of this paper is to contrast and compare the now well-developed theory of Hele-Shaw flow with that of Stokes flow. The second main theme is a description of the theory for Stokes flows, with several new extensions.

Hele-Shaw free surface flows have been the subject of more than 500 papers in the 50 years since the early work of Polubarinova-Kochina [36] and Galin [16]. Stokes flows with 
free surfaces, on the other hand, have until recently received much less attention, but there has been much more activity following the demonstration by Hopper [20, 21] and Richardson [41] that explicit unsteady solutions could be constructed (steady solutions were given earlier in $[\mathbf{4}, \mathbf{1 7}, \mathbf{3 7}, \mathbf{3 9}, \mathbf{4 9}]$ ). A bibliography listing more than 600 papers in both areas can be found at the web address http://www.maths.ox.ac/ 〜howison/ .

We begin by relating the models for the two flows to complex variable theory. We then outline the structure of the two problems, which especially for Stokes flow is somewhat complicated. In later sections we fill in the details of this framework, illustrating it with specific examples.

\section{Hele-Shaw flow: an overview}

\subsection{Formulation}

The Hele-Shaw model (see for instance [33]) is a simple description either of the flow of a viscous Newtonian liquid between two horizontal plates separated by a thin gap, or of a viscous liquid moving under Darcy's law in a porous medium. In a typical situation the fluid occupies a domain whose plan view is $\Omega(t)$ in the $(x, y)$-plane with free boundary $\partial \Omega(t)$; this may be a finite simply-connected blob, the exterior of a finite or infinite inviscid bubble, or more complicated still. The motion is driven by singularities such as sources, sinks or multipoles within the fluid region (and possibly at infinity). We shall primarily consider the case of a single point singularity, usually a source or sink, at the origin.

The fluid velocity averaged across the gap is $\mathbf{u}=\left(u_{1}, u_{2}\right)=-\nabla p(x, y, t)$, where $p$ is the pressure, and for an incompressible fluid

$$
\nabla^{2} p=0 \quad \text { in } \Omega(t),
$$

away from singularities (with appropriate behaviour holding at the singularities) together with the dynamic boundary condition

$$
\text { (a) } p=0 \quad \text { or } \quad \text { (b) } p=\gamma \kappa \text { on } \partial \Omega(t) \text {, }
$$

and the kinematic boundary condition

$$
-\frac{\partial p}{\partial n}=V_{n} \quad \text { on } \partial \Omega(t)
$$

where $\partial / \partial n$ denotes the derivative in the direction of the outward normal $\mathbf{n}$ to $\partial \Omega(t)$, and $V_{n}$ is the velocity of $\partial \Omega(t)$ in the direction of $\mathbf{n}$. Condition (2.1) (a) neglects surface tension effects; in (2.1) (b), $\gamma$ is a dimensionless surface tension coefficient and $\kappa$ the curvature of the free boundary, positive when the fluid domain is convex (but see for instance [35] for more detailed discussion of the appropriate dynamic boundary condition). The Hele-Shaw problem with $\gamma>0$ is notoriously difficult, and in this paper we neglect surface tension effects, assuming condition (2.1) (a) - the zero surface tension (ZST) problem. The negative pressure $-p$ is a velocity potential for the flow; hence if $z$ denotes the complex variable $x+i y$ there is an analytic complex potential $w(z, t)$ for the flow, such that

$$
w(z, t)=-p+i \psi
$$




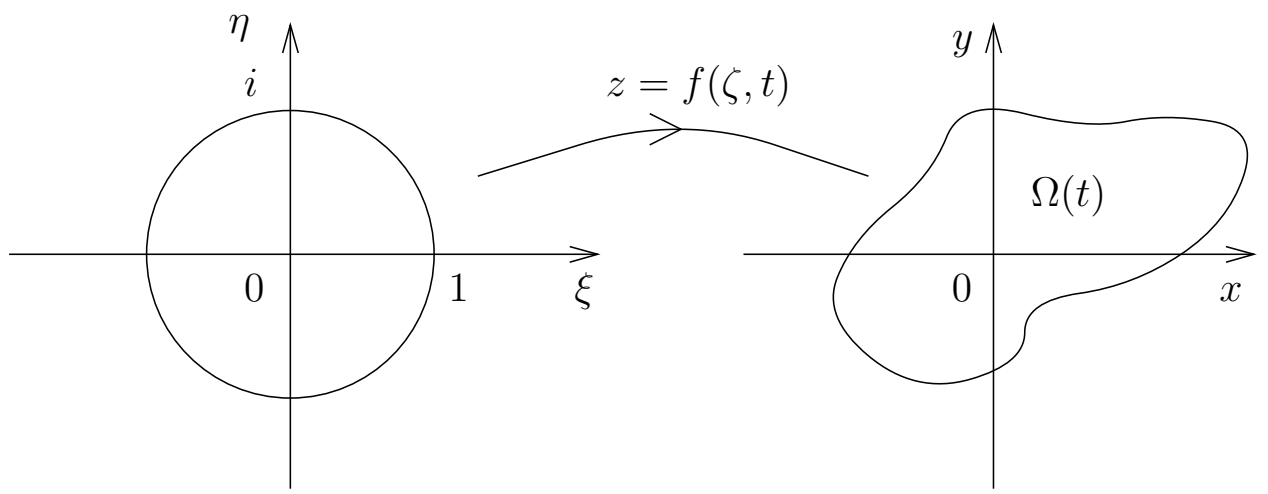

Figure 1. Mapping from the unit disc to $\Omega(t)$.

where $\psi$ is a streamfunction, so that $\left(u_{1}, u_{2}\right)=(\partial \psi / \partial y,-\partial \psi / \partial x)$.

Rather than solving directly, we consider a time-dependent univalent mapping $z=$ $f(\zeta, t)$ between $\Omega(t)$ and the unit disc $|\zeta| \leq 1$, such that the driving singularities of the flow correspond to specified points; if there is only one singularity this is taken to lie either at the origin or at infinity in the $z$-plane, and is made to correspond to $\zeta=0$. Figure 1 shows the configuration. Because $\Re(w)=0$ on $\partial \Omega(t)$, the $\zeta$-plane is essentially a potential plane for the problem. It is thus usually easy to calculate the complex potential in the $\zeta$-plane, $W(\zeta, t)$. The boundary conditions (2.1) and (2.2) then give

$$
\Re\left(\frac{f_{t}(\zeta, t)}{\zeta f^{\prime}(\zeta, t)}\right)=\frac{1}{\left|f^{\prime}(\zeta, t)\right|^{2}} \Re\left(\zeta W^{\prime}(\zeta, t)\right), \quad \text { on }|\zeta|=1,
$$

(when it is unambiguous we write $f^{\prime}=\partial f / \partial \zeta$ and $f_{t}=\partial f / \partial t$, and similarly for other functions and variables). This is known as the Polubarinova-Galin (P-G) equation [36, 16]. Probably the simplest specific example is for flow driven by a single point source (or sink) of strength $Q>0(Q<0)$ at the origin, in which case $W(\zeta) \equiv(Q / 2 \pi) \log \zeta$, and (2.3) becomes

$$
\Re\left(\zeta f^{\prime}(\zeta, t) \bar{f}_{t}(1 / \zeta, t)\right)=\frac{Q}{2 \pi} \quad \text { on }|\zeta|=1 .
$$

The salient points of the theory are summarised in the following.

\subsection{Explicit solutions}

Any rational univalent function $f(\zeta, t)$ gives a solution of (2.4). We illustrate the application of the P-G equation (2.4) for a simple nontrivial mapping function, the polynomial

$$
z=f(\zeta, t)=a(t)\left(\zeta-\frac{b(t)}{n} \zeta^{n}\right),
$$

for integers $n \geq 2$. By suitable choice of co-ordinates we may assume both $a(0)$ and $b(0)$ to be real and positive without loss of generality, a property which persists for $t>0$. The initial map is univalent if and only if $|b(0)|<1$.

Substituting from (2.5) in (2.4) with $\zeta=e^{i \theta}$ and equating coefficients appropriately, 
we obtain the system of ordinary differential equations

$$
\begin{aligned}
& a \frac{d a}{d t}+\frac{a b}{n} \frac{d}{d t}(a b)=\frac{d}{d t}\left[a^{2}\left(1+\frac{b^{2}}{n}\right)\right]=\frac{Q}{\pi}, \\
& \frac{a}{n} \frac{d}{d t}(a b)+a b \frac{d a}{d t}=\frac{1}{n a^{n-1}} \frac{d}{d t}\left(a^{n+1} b\right)=0 .
\end{aligned}
$$

These equations give the evolution until such time as all the fluid is extracted (with $a(t)=0$ ), or until the solution breaks down with loss of univalency of the map (2.5) on the unit disc. Solution breakdown is discussed further in $\S 2.7$.

\subsection{The Schwarz function}

An alternative to the 'brute force' approach of substitution into (2.3), thereby determining the ordinary differential equations satisfied by the the parameters of the map, is to use the Schwarz function. We recall that any analytic curve in the $(x, y)$-plane may be described in terms of a Schwarz function $g(z, t)$, analytic in some neighbourhood of the curve, such that the relation $\bar{z}=g(z, t)$ defines the curve (see [11] for a discussion). The Schwarz function is obtainable from the Cartesian equation of the curve by substituting $x=(z+\bar{z}) / 2, y=(z-\bar{z}) /(2 i)$, and solving for $\bar{z}$.

The complex variable theory we use for both Hele-Shaw and Stokes flow assumes analyticity of the free boundary $\partial \Omega(t)$, which is hence describable by a time-dependent Schwarz function $g(z, t)$. This Schwarz function is related to the conformal map $f(\zeta, t)$ by

$$
g(z, t)=\overline{f(1 / \bar{\zeta}, t)} \equiv \bar{f}(1 / \zeta, t) ;
$$

the second equality here defines the complex conjugate function $\bar{f}$. For the ZST HeleShaw problem, the Schwarz function is related to the complex potential $w(z, t)$ by the global equation

$$
\frac{\partial w}{\partial z}=\frac{1}{2} \frac{\partial g}{\partial t} .
$$

This equation is obtained by differentiating the complex potential with respect to arclength $s$ along the free boundary and using the facts that

$$
\frac{\partial z}{\partial s}=\left(g^{\prime}\right)^{-1 / 2}, \quad V_{n}=-\frac{i}{2} \frac{g_{t}}{\left(g^{\prime}\right)^{1 / 2}} .
$$

Then along $\partial \Omega(t)$,

$$
\frac{\partial w}{\partial z}=\frac{\partial w}{\partial s} / \frac{\partial z}{\partial s}=-\left(\frac{\partial p}{\partial s}+i \frac{\partial p}{\partial n}\right) / \frac{\partial z}{\partial s}=-i\left(g^{\prime}\right)^{1 / 2}\left(-V_{n}\right)=\frac{1}{2} \frac{\partial g}{\partial t} .
$$

As it stands, this identity, first stated in [31], holds only on the free boundary; however since both sides are analytic functions of $z$ in some neighbourhood of $\partial \Omega(t)$, it may be analytically continued away from $\partial \Omega(t)$, and must hold wherever the various quantities are defined. ${ }^{1}$

1 A NZST version of equation (2.9) may also be obtained, using boundary condition (2.1)(b) instead of (2.1)(a), together with an expression for the curvature in terms of the Schwarz function 
The only singularities of $w(z, t)$ within $\Omega(t)$ are the fixed driving (pressure) singularities of the flow, which are prescribed as part of the problem; $g(z, 0)$ is also fixed by the initial data. Hence the singularities of $g(z, t)$ within $\Omega(t)$ evolve in an entirely predictable manner. These singularities are the constant initial singularities of $g(z, 0)$, plus the time integrals of the driving singularities, as indicated by (2.9). In particular, the singularities of $g(z, t)$ within $\Omega(t)$ must remain fixed in space $[\mathbf{3 1}]$.

For a single point source at the origin, $\partial w / \partial z$ has only a simple pole at $z=0$, with residue $Q /(2 \pi)$, and so from (2.9) the time derivative of the Schwarz function has the Laurent expansion

$$
\frac{\partial g}{\partial t}=\frac{Q}{\pi z}+(\text { analytic power series })
$$

Together with (2.8), this affords an alternative method of solving the ZST problem. Equation (2.8) is used to obtain the Laurent expansion of $g(z, t)$ about $z=0$, then matching coefficients in (2.10) gives equations determining the parameters in the map. To illustrate, with the mapping function (2.5), equation (2.8) gives

$$
g(f(\zeta, t), t)=\frac{a(t)}{\zeta}-\frac{a(t) b(t)}{n \zeta^{n}} .
$$

Inversion of the map near the origin gives the local form of the Schwarz function as

$$
g(z, t)=-\frac{a^{n+1} b}{n} \frac{1}{z^{n}}+a^{2}\left(1+\frac{b^{2}}{n}\right) \frac{1}{z}+O(1) \quad \text { near } z=0 .
$$

Hence from (2.10) we find

$$
\begin{aligned}
\frac{d}{d t}\left(a^{n+1} b\right) & =0, \\
\frac{d}{d t}\left(a^{2}\left(1+\frac{b^{2}}{n}\right)\right) & =\frac{Q}{\pi},
\end{aligned}
$$

exactly as obtained in $\S 2.2$ by different means. Such results are readily extended to the case of flow driven by many sources or sinks [40], or even through slits [42].

The Schwarz function is closely linked to the Cauchy transform $\vartheta(z, t)$ of the fluid domain $[40,42,43]$. This is defined as

$$
\vartheta(z, t)=-\frac{1}{\pi} \iint_{\Omega(t)} \frac{d x^{\prime} d y^{\prime}}{z^{\prime}-z}
$$

where $z^{\prime}=x^{\prime}+i y^{\prime}$. It is a useful tool for dealing with multiply-connected fluid domains [43], although as we just assume a bounded, simply-connected fluid domain we shall not exploit its potential fully. The right-hand side of (2.13) defines a function of $z$, denoted by $\vartheta_{e}(z, t)$, which is analytic for $z$ exterior to $\Omega(t)$. This function may be analytically continued inside $\Omega(t)$, but this continuation will in general have singularities. In [42] it (also given in $[\mathbf{1 1}]$ ). The equation is

$$
\frac{\partial w}{\partial z}=\frac{1}{2} \frac{\partial g}{\partial t}-\frac{i \gamma}{2} \frac{\partial}{\partial z} \frac{g^{\prime \prime}}{\left(g^{\prime}\right)^{3 / 2}}
$$

(see $[\mathbf{2 4}]$ ), which illustrates the difficulty of the NZST problem compared with the ZST. 
is shown that

$$
\vartheta(z, t)= \begin{cases}\vartheta_{e}(z, t) & \text { for } z \text { outside } \Omega(t), \\ \bar{z}-\vartheta_{i}(z, t) & \text { for } z \text { inside } \Omega(t),\end{cases}
$$

where $\vartheta_{i}$ is analytic inside $\Omega(t)$. From the definition (2.13), $\vartheta$ is clearly continuous throughout the $(x, y)$-plane, and hence on $\partial \Omega(t)$,

$$
\bar{z}=\vartheta_{e}(z, t)+\vartheta_{i}(z, t)
$$

However, the right-hand side here must also be equal to the Schwarz function $g(z, t)$ on the free boundary, by definition. Hence, analytically continuing, the above provides a decomposition of $g(z, t)$ into parts analytic exterior to and inside $\Omega(t)$ respectively $\left(g_{e}(z, t) \equiv \vartheta_{e}(z, t)\right.$ and $\left.g_{i}(z, t) \equiv \vartheta_{i}(z, t)\right)$, which is unique if we insist $g_{e}(z, t) \rightarrow 0$ as $z \rightarrow \infty$.

\subsection{The Baiocchi transformation}

A third way of looking at the Hele-Shaw problem is prompted by the desire to construct a weak formulation in terms of an elliptic variational inequality. As it stands this is not possible, because the boundary conditions are not suitable. However, integration in time obviates this difficulty, and the resulting transform of the pressure is often known as the Baiocchi transform [3]; it was first used for the Hele-Shaw problem in $[\mathbf{1 3}]$. Here, we adopt a slightly different approach. We write down the equation and boundary conditions satisfied by the transformed variable, which we call $u(x, y, t)$, and then seek to relate $u$ to a physical variable. The first step of this procedure can be carried out for any free boundary problem, but the second is clearly more problematic.

Consider then a quite general free boundary problem on a domain $\Omega(t)$, with boundary $\partial \Omega(t)$ described by the Schwarz function $g(z, t)$. The analyticity of $g$ enables us to write $g(z, t)=h^{\prime}(z, t)$ for some $h$ analytic in the same region as $g$. Define the real variable $u$ by the formula

$$
u(z, \bar{z}, t)=\frac{1}{4}(z \bar{z}-h(z, t)-\overline{h(z, t)})
$$

(note that, while $h$ is multivalued, its real part is not). It is clear that $u$ satisfies the Poisson equation $\nabla^{2} u=1$ and, since $\bar{z}=g(z, t)$ on $\partial \Omega(t)$, the boundary conditions

$$
\frac{\partial u}{\partial z}=0=\frac{\partial u}{\partial \bar{z}} \quad \text { on } \partial \Omega(t)
$$

Choosing the arbitrary function of time in $h(z, t)$ appropriately, $u$ satisfies the following Cauchy problem:

$$
\begin{gathered}
\nabla^{2} u=1 \quad \text { in } \Omega(t), \\
u=0=\frac{\partial u}{\partial n} \quad \text { on } \partial \Omega(t) .
\end{gathered}
$$

Conversely, if $u$ is the solution of this Cauchy problem and the function $\widetilde{g}$ is defined by

$$
\widetilde{g}=\bar{z}-2\left(\frac{\partial u}{\partial x}-i \frac{\partial u}{\partial y}\right)
$$


then $\widetilde{g}$ is an analytic function of $z$, as its real and imaginary parts satisfy the CauchyRiemann equations; furthermore, $\widetilde{g}=\bar{z}$ on $\partial \Omega(t)$. It follows that $\widetilde{g}(z, t) \equiv g(z, t)$, and we have recovered the Schwarz function of the free boundary.

We now restrict ourselves to the specific case of ZST Hele-Shaw flow. Differentiating the definition (2.14) gives

$$
\frac{\partial^{2} u}{\partial t \partial z}=-\frac{1}{4} \frac{\partial^{2} h}{\partial t \partial z}=-\frac{1}{4} \frac{\partial g}{\partial t}=-\frac{1}{2} \frac{\partial w}{\partial z}
$$

using (2.9). Integrating with respect to $z$, recalling that $u$ is real and has been chosen to vanish on $\partial \Omega(t)$, and that the pressure is the real part of the complex potential $w$, we find the well-known relation

$$
\frac{\partial u}{\partial t}=p
$$

As mentioned above, the 'Baiocchi variable' $u$ for the Hele-Shaw problem is usually introduced via the time integral of the pressure (see for example $[\mathbf{3}, \mathbf{1 3}, \mathbf{3 0}, \mathbf{3 1}]$ ), though care is needed with the exact definitions depending on whether one is dealing with injection $(p>0)$ or suction $(p<0)$.

The additional imposition of the constraint $u \geq 0$ leads to a variational inequality for $u$, and a characterisation of 'well-posed' Hele-Shaw problems as time derivatives of one-parameter families of variational inequalities. This approach is clearly possible in any number of space dimensions; a complex variable approach necessarily limits the dimension to two. As an aside we note that, with this additional requirement $u \geq 0,(2.15)$ and (2.16) are together equivalent to the obstacle problem of variational calculus, ${ }^{2}$ hence in certain circumstances properties of solutions to the Hele-Shaw free boundary problem can be inferred from known results for this problem. An example is the classification of the possible transient cusps in a Hele-Shaw flow [23].

These considerations suggest another approach to the problem. One first solves the (ill-posed) Cauchy problem for $u_{0}(z, \bar{z}) \equiv u(z, \bar{z}, 0)$,

$$
\nabla^{2} u_{0}=1 \quad \text { in } \Omega(0), \quad u_{0}=0=\frac{\partial u_{0}}{\partial n} \quad \text { on } \partial \Omega(0) .
$$

This, together with the relation $\partial u / \partial t=p$, gives a complete description of the interior singularities of $u(z, \bar{z}, t)$. They are exactly the singularities of $u_{0}(z, \bar{z})$ within $\Omega(0)$, plus the time integrals of the driving pressure singularities. Finally, one must solve the free boundary problem (2.15), (2.16) for $u(z, \bar{z}, t)$, with these singularities.

\subsection{Moments and conserved quantities}

As indicated above, a univalent rational map with a finite number of time-dependent parameters gives an explicit solution to the ZST Hele-Shaw problem. The solutions of the resulting ordinary differential equations for the parameters have an attractive geometric interpretation in terms of certain moments of the domain $\Omega(t)$.

2 This is the (well-posed) problem of determining the contact region when a membrane is stretched over a smooth obstacle, so that it is in contact with only part of the obstacle (see for instance [14]). The Baiocchi variable $u$ represents the membrane displacement. 
For a flow driven by a single point source of constant strength $Q>0$ at the origin, the pressure $p$ satisfies the distributional equation

$$
\nabla^{2} p=-Q \delta(x) \delta(y) \quad \text { in } \Omega(t)
$$

For any function $L(z)$ analytic on $\Omega(t)$, use of Green's theorem shows that [40]

$$
\frac{d}{d t} \iint_{\Omega(t)} L(z) d x d y=\int_{\partial \Omega(t)} L(z) V_{n} d s=-\int_{\partial \Omega(t)} L(z) \frac{\partial p}{\partial n} d s=Q L(0) .
$$

In particular, taking the integrand $L(z)=z^{k}$ for positive integers $k$ we obtain the infinite set of moments $M_{k}(t), k=0,1, \ldots$, which satisfy

$$
\frac{d M_{k}(t)}{d t} \equiv \frac{d}{d t} \iint_{\Omega(t)} z^{k} d x d y=Q \delta_{0 k}
$$

Thus, all the moments are constant except the area $(k=0)$, which changes at the rate $Q$. This result (2.18) generalises easily to the case of a system of sources/sinks within $\Omega(t)[\mathbf{4 0}]$, or to multipole singularities [15].

The moment evolution is also easy to obtain via the Baiocchi transform. For the point source problem, we have

$$
\nabla^{2} u=-Q t \delta(x) \delta(y)+1 \quad \text { in } \Omega(t)
$$

the identity

$$
\iint_{\Omega(t)}\left(z^{k} \nabla^{2} u-u \nabla^{2}\left(z^{k}\right)\right) d x d y=\int_{\partial \Omega(t)}\left(z^{k} \frac{\partial u}{\partial n}-u \frac{\partial z^{k}}{\partial n}\right) d s
$$

immediately leads to

$$
\iint_{\Omega(t)} z^{k} d x d y=Q t \delta_{0 k}+\{\text { time-independent }\}
$$

whence follows (2.18).

For the example of the polynomial map (2.5), the only nonzero moments are the area $M_{0}$, and $M_{n-1}$. Using the definition in (2.18) and transferring the integrals to the $\zeta$-plane we find

$$
M_{0}(t)=\pi a^{2}(t)\left(1+\frac{b^{2}(t)}{n}\right), \quad M_{n-1}(t)=-\frac{\pi a^{n+1}(t) b(t)}{n} .
$$

With equation (2.18), this gives the same as (2.6) and (2.7) obtained using the P-G equation (or (2.11) and (2.12) obtained using the Schwarz function).

The results of this section are directly linked to the singular behaviour of the Schwarz function (§2.3). Using Green's theorem (in complex form) on the definition of $M_{k}(t)$,

$$
M_{k}(t)=\iint_{\Omega(t)} z^{k} d x d y=\frac{1}{2 i} \int_{\partial \Omega(t)} \bar{z} z^{k} d z \equiv \frac{1}{2 i} \int_{\partial \Omega(t)} g(z, t) z^{k} d z .
$$

Hence,

$$
\begin{aligned}
\frac{d M_{k}}{d t} & =\frac{1}{2 i} \int_{\partial \Omega(t)}\left(\frac{\partial}{\partial t}\left(g(z, t) z^{k}\right)+\frac{\partial}{\partial z}\left(g(z, t) z^{k} V_{n}\right)\right) d z \\
& =\frac{1}{2 i} \int_{\partial \Omega(t)} \frac{\partial g}{\partial t} z^{k} d z
\end{aligned}
$$


and the moment-evolution equations follow immediately (for the specific case of point source-driven flow) from (2.10) of §2.3. In particular, the above shows that the quantities $d M_{k} / d t$ are the coefficients of the principal part of the Laurent expansion for $\pi \partial g / \partial t$ about the origin. Alternatively, recalling the comments at the end of $\S 2.3$, we have the following relations for the singular parts of the Schwarz function and Cauchy transform:

$$
g_{e}(z, t)=\vartheta_{e}(z, t)=\frac{1}{\pi} \sum_{k=0}^{\infty} \frac{M_{k}(t)}{z^{k+1}},
$$

for $|z|$ sufficiently large that this sum converges.

\subsection{A quadrupole in a circle}

In this section we outline a deductive procedure (due to Richardson [38]) for finding the form of the mapping function $f(\zeta, t)$ for a particular ZST Hele-Shaw initial-value problem. We illustrate the procedure by solving for a quadrupole singularity placed at the centre of an initially-circular fluid domain. ${ }^{3}$ This problem was solved by Entov et al. [15], using the moments approach rather than the Schwarz function method we use here; note that steady-state solutions of the NZST problem are also constructed in [15] using a Schwarz function approach.

The method relies on equation (2.9). For a quadrupole singularity of strength $M>0$ at the origin the complex potential $w(z, t)$ has only one singularity:

$$
w(z, t)=-\frac{M}{z^{2}}+O(1) \quad \text { as } z \rightarrow 0,
$$

thus by (2.9) the Schwarz function has the local behaviour

$$
\frac{\partial g}{\partial t}=\frac{4 M}{z^{3}}+O(1) \quad \text { as } z \rightarrow 0 .
$$

Decomposing the Schwarz function into parts regular and singular within the fluid domain as in $§ 2.3$, the singular part must satisfy

$$
g_{e}(z, t)=g_{e}(z, 0)+\frac{4 M t}{z^{3}} .
$$

The Schwarz function of the initial domain (a circle of radius $r$ centred on the origin) is

$$
g(z, 0)=\frac{r^{2}}{z} \equiv g_{e}(z, 0),
$$

hence

$$
g_{e}(z, t)=\frac{r^{2}}{z}+\frac{4 M t}{z^{3}} .
$$

Since the origin in the $\zeta$-plane maps to the origin in the $z$-plane, equation (2.8) implies that the function $\bar{f}(1 / \zeta, t)$ has a triple pole at $\zeta=0$ and no other singularities within

3 While there is no special reason for this choice of example here, there is a good reason to choose it to illustrate the analogous Stokes flow procedure of $\S 3.6$, and our desire is to keep the discussion of the two problems as parallel as possible. 
the unit disc; moreover, it must vanish at infinity. It follows that $\bar{f}(1 / \zeta, t)$ is of the form

$$
\bar{f}(1 / \zeta, t)=\frac{a(t)}{\zeta}+\frac{b(t)}{\zeta^{2}}+\frac{c(t)}{\zeta^{3}},
$$

for some functions $a(t), b(t), c(t)$. From the symmetry of the initial domain and driving mechanism, $\Omega(t)$ remains symmetric about the $x$-axis, whence $a(t)$ and $c(t)$ are real and $b(t) \equiv 0$. Hence

$$
f(\zeta, t)=a(t) \zeta+c(t) \zeta^{3} .
$$

From here the solution procedure follows the example of $\S 2.3$. A local inversion of the map near the origin gives the local behaviour of $g(z, t)$ as

$$
g(z, t)=\frac{a^{3} c}{z^{3}}+\frac{1}{z}\left(a^{2}+3 c^{2}\right)+O(1),
$$

thus using (2.20) we find

$$
\begin{aligned}
\frac{d}{d t}\left(a^{2}+3 c^{2}\right) & =0, \\
\frac{d}{d t}\left(a^{3} c\right) & =4 M .
\end{aligned}
$$

Taking $a(0)=1, a(t)$ satisfies

$$
a^{8}(t)-a^{6}(t)+48 M^{2} t^{2}=0
$$

with

$$
c(t)=\frac{4 M t}{a^{3}(t)} .
$$

The evolution is shown in Figure 2. The moving boundary changes smoothly from its initial circular form until time $t^{*}=3 /(64 M)$ at which point $a\left(t^{*}\right)=\sqrt{3} / 2$, and the solution blows up with the simultaneous formation of two cusps in the free boundary.

\subsection{Blow-up}

We have just given an example of singularity formation for ZST Hele-Shaw flows. We are not in this paper primarily concerned with blow-up, but rather with the underlying mathematical structure of the free boundary problems. Nevertheless, blow-up is such an important feature of the ZST Hele-Shaw problem that we discuss it briefly.

ZST Hele-Shaw problems driven by a single point source/sink correspond to 'injection'/'suction' respectively. Injection problems have pressure $p>0$ everywhere, and the free boundary advances; suction problems have $p<0$ everywhere, and the free boundary retreats. ${ }^{4}$ It is well-known that the suction problem in a finite domain is ill-posed, and its solutions undergo finite-time blow-up in all but very special cases. We have already

${ }^{4}$ We can classify some problems in this manner using the maximum principle. although for fluid domains containing sources and sinks, or more general driving mechanisms, the maximum principle cannot necessarily be applied in the same way. There is no maximum principle for the biharmonic equation, so the advancing/retreating question for Stokes flow is potentially more complicated even when the driving mechanism is simple. 


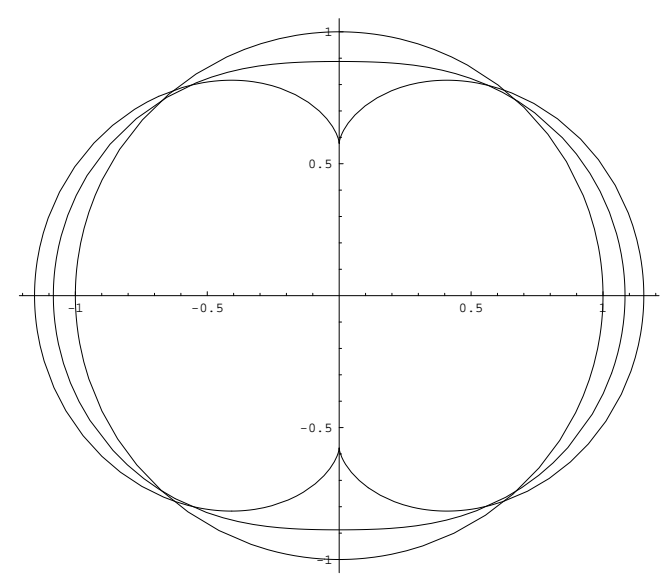

FIGURE 2. Evolution of an initially circular region containing a quadrupole, up to the time of cusp formation.

seen an instance of finite-time blow-up in the simple example of the polynomial mapping function (2.5), with the evolution determined by equations (2.6) and (2.7). When $Q>0$ (the injection problem) this proceeds smoothly and the free boundary approaches a large expanding circle as $t \rightarrow \infty$; however in the suction problem with $Q<0$ solution breakdown occurs at a time $t^{*}$ before all the fluid is removed. We find $b\left(t^{*}\right)=1$ and $a\left(t^{*}\right)>0$, so that $f^{\prime}\left(\zeta, t^{*}\right)$ has $n-1$ zeroes on $|\zeta|=1$; the map (2.5) loses univalency via the simultaneous formation of $n-1$ symmetrically placed cusps of $3 / 2$-power in the free boundary. In the simplest case $n=2$ the free boundary is initially a limaçon, evolving into a cardioid at time $t^{*}$.

It can be shown that, where the fluid domain is described by a polynomial mapping function, cusp formation is the generic form of blow-up via loss of analyticity of the free boundary [18]. In almost all cases the $\operatorname{cusp}(\mathrm{s})$ formed at blow-up time are of 3/2-power type, although other types of cusp can also occur (of power $5 / 2,7 / 2, \ldots$ etc.). Blow-up is also possible with the free boundary remaining analytic, by self-overlapping of the free boundary, and in more general problems by the formation of other types of singularity in the free boundary such as corners $[\mathbf{2 9}, \mathbf{3 0}]$. For the case of a general mapping function it is difficult to be specific about the exact manner of blow-up (though see for example $[\mathbf{1 8}, \mathbf{4 5}]$, where various possibilities are catalogued).

Special cases are blow-up via cusps of power $(4 n+1) / 2$ for integer $n$; it can be shown [47] that the obstacle problem admits solutions with singularities of this and only this type in its free boundary, and likewise explicit Hele-Shaw solutions can be found in which the free boundary forms a 5/2-power cusp; having formed, it immediately disappears, 
and the evolution continues smoothly apart from this instantaneous singularity. By a time-reversal argument, such behaviour must necessarily be observable for both suction and blowing problems.

General blowing problems are well-posed locally in time and, apart from special cases such as the above (and of course blow-up by overlapping of the free boundary, which is always possible), solutions with smooth initial data are well-behaved globally. In many cases, even solutions with nonanalytic data (e.g. cusps in $\partial \Omega(0)$ ) are well-behaved, with the free boundary smoothing immediately, and until recently it was conjectured that this was generic. However, King et al. $[\mathbf{2 9}]$ demonstrated that blowing problems with an acute-angled corner in $\partial \Omega(0)$ can exhibit waiting time behaviour, with the corner persisting for some finite waiting time $t_{w}$, after which the corner angle jumps abruptly to the supplementary value, and evolution thereafter proceeds smoothly.

\section{Stokes flow: an overview}

\subsection{Formulation}

We use the same notation as introduced for the Hele-Shaw problem in $\S 2$. The slow flow equations in the absence of gravity are

$$
\nabla p=\mu \nabla^{2} \mathbf{u}, \quad \nabla \cdot \mathbf{u}=0
$$

(see for instance $[\mathbf{3 2}]$ ), where $\mu$ denotes the fluid viscosity. We mostly consider flows in which gravitational effects are negligible, although they are briefly mentioned in $\S 5.1$. With nonzero gravity one has to define a 'reduced pressure' to account for its effect, which leads to modified (more difficult) boundary conditions for the time-dependent problem, and the procedures of analytic continuation described below do not follow through (see also $\S 5.1$ below). Garabedian [17] has considered some steady Stokes flows with gravity.

For the two-dimensional problem there is again a streamfunction $\psi(x, y, t)$ which, away from driving singularities, satisfies the biharmonic equation

$$
\nabla^{4} \psi=0 \quad \text { in } \Omega(t) .
$$

In addition there is an Airy stress function $\mathcal{A}(x, y, t)$, also biharmonic, related to the Newtonian stress tensor $\sigma_{i j}$ by

$$
\begin{aligned}
& \sigma_{11}=-p+2 \mu \partial u_{1} / \partial x=-2 \mu \partial^{2} \mathcal{A} / \partial y^{2} \\
& \sigma_{12}=\sigma_{21}=\mu\left(\partial u_{1} / \partial y+\partial u_{2} / \partial x\right)=2 \mu \partial^{2} \mathcal{A} / \partial x \partial y, \\
& \sigma_{22}=-p+2 \mu \partial u_{2} / \partial y=-2 \mu \partial^{2} \mathcal{A} / \partial x^{2} .
\end{aligned}
$$

It follows from these equations that $p=\mu \nabla^{2} \mathcal{A}$. Also, the vorticity $\omega=-\nabla^{2} \psi$ so the combination $p / \mu+i \omega$ is an analytic function. As well as the usual kinematic boundary condition there are two stress boundary conditions on $\partial \Omega(t)$,

$$
\sigma_{i j} n_{j}=-\gamma \kappa n_{i} \quad i=1,2
$$

where $\mathbf{n}=\left(n_{i}\right)$ denotes the outward normal to $\partial \Omega(t)$. These stress boundary conditions take the simplest form when written in terms of $\mathcal{A}$; it can be shown (see for instance $[\mathbf{2 8}]$ ) 
that if arbitrary constants of integration are chosen appropriately, then (3.2) implies

$$
\mathcal{A}=0, \quad \frac{\partial \mathcal{A}}{\partial n}=\frac{\gamma}{2 \mu} \quad \text { on } \partial \Omega(t)
$$

The kinematic boundary condition, however, is best written in terms of $\psi$, giving

$$
\frac{\partial \psi}{\partial s}=-V_{n} \quad \text { on } \partial \Omega(t)
$$

We note from equation (3.3) that, unlike the Hele-Shaw case, the addition of surface tension effects does not constitute a singular perturbation as $\gamma \rightarrow 0$.

Using the Goursat representation of biharmonic functions $\psi$ and $\mathcal{A}$ may be expressed in the form

$$
\mathcal{A}+i \psi=\mathcal{W}(z, \bar{z}, t)=-(\bar{z} \phi(z, t)+\chi(z, t))
$$

for functions $\phi(z, t), \chi(z, t)$ analytic on $\Omega(t)$ except at driving singularities. ${ }^{5}$ As for HeleShaw, if there is only one such singularity this is taken to lie at the origin (or at infinity). All physical quantities of interest may be expressed in terms of the Goursat functions $\phi$ and $\chi$; for instance the pressure and velocity fields are given by

$$
\begin{aligned}
p & =-4 \mu \Re\left(\phi^{\prime}(z, t)\right), \\
u_{1}+i u_{2} & =\phi(z, t)-z \overline{\phi^{\prime}(z, t)}-\overline{\chi^{\prime}(z, t)} .
\end{aligned}
$$

In terms of these functions, the conditions (3.2) are easily seen to give the single complex boundary condition $[\mathbf{3 7}]$

$$
\phi(z, t)+z \overline{\phi^{\prime}(z, t)}+\overline{\chi^{\prime}(z, t)}=\frac{i \gamma}{2 \mu} \frac{d z}{d s} \quad \text { on } \partial \Omega(t)
$$

Following the development of the Hele-Shaw problem, we map the unit disc $|\zeta|<1$ onto $\Omega(t)$. Again, we consider the case of just one driving singularity, and we write $z=f(\zeta, t)$, where $f(0, t)=0$. The analytic functions $\phi(z, t), \chi(z, t)$ then correspond to functions $\Phi(\zeta, t), \mathcal{X}(\zeta, t)$, themselves analytic (on the unit disc) away from the singularity at $\zeta=0$. An important difference now emerges from the corresponding treatment for Hele-Shaw. There, specification of the singular part of the complex potential $w(z, t)$ at the sink $z=0$, equivalent to specifying the singular part of its Laurent expansion, was enough to determine $w(z, t)$ completely. Here, on physical grounds we specify the singular parts of $\phi(z, t)$ and $\chi(z, t)$, but now this is not enough to determine these functions uniquely. More precisely, the $O(1)$ term in $\phi(z, t)$ remains free, and this means that the velocity field (3.6) is only specified up to the addition of uniform translation by $\phi(0, t)$. In other words, the velocity at the origin consists of the singular part, plus an undetermined uniform stream (see also [25]). This degeneracy should be expected, as a rigid-body motion, even unsteady, is automatically a solution of the Stokes equations and boundary conditions. We therefore consider how it might be removed. We can for example, as was done in $[\mathbf{4 1}]$, insist that $\phi(0, t)=0$ (or, although we do not yet do so, any other specified

$5 \mathcal{A}$ and $\psi$ are 'biharmonic conjugates'; their 'Cauchy-Riemann' equations are

$$
\partial^{2} \mathcal{A} / \partial x^{2}-\partial^{2} \mathcal{A} / \partial y^{2}=2 \partial^{2} \psi / \partial x \partial y \quad \text { and } \quad \partial^{2} \psi / \partial x^{2}-\partial^{2} \psi / \partial y^{2}=-2 \partial^{2} \mathcal{A} / \partial x \partial y
$$


function of $t$ ). This purely mathematical assumption is clearly physically appropriate in cases where the flow is symmetric about, for example, the $x$ - and $y$-axes, for then in the absence of an externally-imposed uniform translation the non-singular velocity at $z=0$ vanishes. If, however, we impose $\phi(0, t)=0$ in other cases, we shall see that although exact solutions can be generated, they do not, in general, conserve overall linear or angular momentum. Although the Stokes system itself does not enforce momentum conservation, it is clearly desirable to try to choose solutions that do, not least as this is the solvability condition for the $O(\mathrm{Re})$ term in a small Reynolds number (Re) expansion of the Navier-Stokes equations. We can impose momentum conservation in one of two ways:

(1) As mentioned above, by insisting that $\phi(0, t)=0$. This leads to attractively simple explicit solutions, but the disadvantage is that we are forced to move the driving singularities in a prescribed and usually unnatural way.

(2) By determining $\phi(0, t)$ from the condition of conservation of overall momentum. Unfortunately this latter approach is technically much more difficult.

In the remainder of Section 3, unless we specifically state otherwise, we impose $\phi(0, t)=0$.

Let us now return to our outline of the theory. Referring to [41] and [10] for the details (though we give a brief outline in $\S 4$ ), when we reformulate the problem in the $\zeta$-plane, the boundary conditions may be analytically continued off the unit circle to give functional identities holding globally in the $\zeta$-plane (equations (2.18) and (2.19) of [41]). In terms of the functions $\mathcal{X}(\zeta, t)$ and $\Phi(\zeta, t)$ introduced above, these equations are most conveniently expressed as follows:

$$
\frac{\partial}{\partial t}\left(f^{\prime}(\zeta, t) \bar{f}(1 / \zeta, t)\right)+2 \mathcal{X}^{\prime}(\zeta, t)=\frac{\gamma}{2 \mu} \frac{\partial}{\partial \zeta}\left(\zeta f^{\prime}(\zeta, t) \bar{f}(1 / \zeta, t) G_{+}(\zeta, t)\right),
$$

and

$$
2 \Phi(\zeta, t)-\frac{\partial f}{\partial t}(\zeta, t)+\frac{\gamma}{2 \mu} G_{+}(\zeta, t) \zeta f^{\prime}(\zeta, t)=0,
$$

where the function $G_{+}(\zeta, t)$ (analytic for $|\zeta|<1$ ) is defined within the unit disc in terms of the conformal map via

$$
G_{+}(\zeta, t)=\frac{1}{2 \pi i} \oint_{|\tau|=1} \frac{1}{\left|f^{\prime}(\tau, t)\right|} \frac{\tau+\zeta}{\tau-\zeta} \frac{d \tau}{\tau} \quad \text { for }|\zeta|<1
$$

(note that its real part is positive). Hence the solution procedure again entails a search for suitable conformal maps $z=f(\zeta, t)$, this time satisfying (3.8) and (3.9).

\subsection{Explicit solutions}

It is a surprising fact that for Stokes flow (unlike Hele-Shaw) we can find singularitydriven solutions with nonzero surface tension coefficient $\gamma$, the nonzero surface tension (NZST) problem. The solution procedure relies on matching singularities within the unit disc on both sides of (3.8), having postulated a particular driving mechanism (manifested as specified singularities in $\mathcal{X}(\zeta, t)$ and $\Phi(\zeta, t))$ and mapping function. 
As for Hele-Shaw, it is easy to check that any rational function gives a solution. However, we now have the important difference that the driving singularity may have to move relative to the fluid. Such relative motion is inevitable if we have more than one driving singularity, or a driving singularity at infinity, ${ }^{6}$ and even for the case of an isolated singularity at the origin it is sometimes unavoidable. To illustrate, and to compare with the Hele-Shaw results, consider the fluid domain described by the polynomial mapping function (2.5), namely $z=f(\zeta, t)=a(t)\left(\zeta-b(t) \zeta^{n} / n\right)$, with $a(0)=a_{0}>0$ and $b(0)=b_{0}$, $0<b_{0}<1$. Assume also that the fluid is driven by a single point source $(Q>0)$ or sink $(Q<0)$ at the origin (this problem was solved in [25]). In terms of the Goursat functions $\phi(z, t)$ and $\chi(z, t)$, this necessitates the local behaviour

$$
\phi(z, t) \text { regular, } \quad \chi(z, t)=-\frac{Q}{2 \pi} \log z+O(1), \quad \text { as } z \rightarrow 0 .
$$

The only singular point of (3.8) within the unit disc is then $\zeta=0$, where the local behaviour must satisfy

$$
\frac{\partial}{\partial t}\left(f^{\prime}(\zeta, t) \bar{f}(1 / \zeta, t)\right)-\frac{\gamma}{2 \mu} \frac{\partial}{\partial \zeta}\left(\zeta f^{\prime}(\zeta, t) \bar{f}(1 / \zeta, t) G_{+}(\zeta, t)\right)=\frac{Q}{\pi \zeta}+O(1)
$$

On the left-hand side here we find

$$
f^{\prime}(\zeta, t) \bar{f}(1 / \zeta, t)=-\frac{a^{2}(t) b(t)}{n \zeta^{n}}+\frac{a^{2}(t)}{\zeta}\left(1+\frac{b^{2}(t)}{n}\right)+O\left(\zeta^{n-2}\right),
$$

and

$$
\zeta f^{\prime}(\zeta, t) \bar{f}(1 / \zeta, t) G_{+}(\zeta, t)=-\frac{a^{2}(t) b(t)}{n \zeta^{n-1}} G_{+}(0, t)+O(1) .
$$

Matching singularities on both sides of (3.8) we thus see that

$$
\frac{d}{d t}\left(a^{2}\left(1+\frac{b^{2}}{n}\right)\right)=\frac{Q}{\pi}, \quad \frac{d}{d t}\left(a^{2} b\right)=-\frac{\gamma}{2 \mu}(n-1) a^{2} b G_{+}(0, t) .
$$

The term $G_{+}(0, t)$ is found from (3.10) to be

$$
G_{+}(0, t)=\frac{2}{\pi a} K(b)
$$

where $K(\cdot)$ denotes the complete elliptic integral of the first kind. The evolution is determined by solving these equations for the coefficients $a$ and $b$. When $\gamma>0$ the solution does not break down for either the injection or the suction case (with $Q>0$ the fluid domain approaches a large expanding circle; with $Q<0$ all fluid is extracted). When $\gamma=0$ the same result holds for $Q>0$, but for $Q<0$ we now have finite time solution breakdown via $(n-1)$ cusps of $3 / 2$-power type, which appear at time $t^{*}=(-\pi / Q) a_{0}^{2}\left(1-b_{0}\right)\left(1-b_{0} / n\right)$. Thus blow-up occurs here as well as as in the HeleShaw problem (we return to this point in $\S 3.7$ ).

The particular solution with $n=2$ is of further interest since it illustrates the drawback warned of above, that if we insist $\phi(0, t)=0$ there is relative motion between the

${ }^{6}$ In this case, the whole fluid domain may be considered as moving with a time-dependent velocity. 
singularity and the fluid domain. The solution procedure ensures that the source/sink remains fixed at the origin; however it is easily checked that $\Omega(t)$ has a nonzero component of momentum along the $x$-axis. In $[\mathbf{2 5}]$ it is given that

$$
\iint_{\Omega(t)}\left(u_{1}+i u_{2}\right) d x d y=\frac{\gamma}{\mu} a^{2} b \frac{1-b^{2}}{2-b^{2}} K(b)+\frac{Q}{2} \frac{a b}{\left(2-b^{2}\right)} .
$$

Subtracting off the appropriate rigid-body motion (Stokes flow being invariant under rigid-body motions) gives an exactly equivalent solution where $\Omega(t)$ has zero net momentum, but the source/sink translates in a specified manner within the fluid.

\subsection{The Schwarz function for ZST Stokes flow}

We saw in $\S 2.3$ how the evolution of a Hele-Shaw flow is intimately linked to the singularities of the Schwarz function within the physical domain $\Omega(t)$. We now ask how (if at all) ZST Stokes flow evolution is related to the singular behaviour of the Schwarz function.

As in $\S 2.4$ we write $g(z, t)=h^{\prime}(z, t)$ for a function $h$ (analytic in the same region as $g$ ), and define $H(\zeta, t)=h(f(\zeta, t), t)$. Since by equation $(2.8) g(z, t)=g(f(\zeta, t), t)=\bar{f}(1 / \zeta, t)$, we have

$$
H^{\prime}(\zeta, t)=\frac{\partial}{\partial \zeta}(h(f(\zeta, t), t))=g(f(\zeta, t), t) f^{\prime}(\zeta, t)=f^{\prime}(\zeta, t) \bar{f}(1 / \zeta, t) .
$$

Equation (3.8) with $\gamma=0$ can then be written in terms of $H(\zeta, t)$ and integrated once with respect to $\zeta$, choosing the arbitrary function of time appropriately, to give

$$
-\mathcal{X}(\zeta, t)=\frac{1}{2} \frac{\partial H(\zeta, t)}{\partial t}
$$

an equation analogous to (2.9) for the Hele-Shaw problem.

Considering the usual example with the mapping function (2.5), we already have (from $\S 2.3)$ the expression for $g(z, t)$ in the neighbourhood of the origin, namely

$$
g(z, t)=-\frac{a^{n+1} b}{n} \frac{1}{z^{n}}+a^{2}\left(1+\frac{b^{2}}{n}\right) \frac{1}{z}+O(1) \quad \text { near } z=0 .
$$

Thus,

$$
h(z, t)=\frac{a^{n+1} b}{n(n-1)} \frac{1}{z^{n-1}}+a^{2}\left(1+\frac{b^{2}}{n}\right) \log z+O(1) \quad \text { near } z=0 .
$$

In terms of $\zeta$ then, (2.5) gives

$$
H(\zeta, t)=\frac{a^{2} b}{n(n-1) \zeta^{n-1}}+a^{2}\left(1+\frac{b^{2}}{n}\right) \log \zeta+O(1) \quad \text { near } \zeta=0 .
$$

The behaviour of $\chi(z, t)$ near the origin is given by (3.11), so in the $\zeta$-plane

$$
\mathcal{X}(\zeta, t)=-\frac{Q}{2 \pi} \log \zeta+O(1) \quad \text { near } \zeta=0 .
$$

Hence matching singularities at the origin in (3.14) we see that $a(t)$ and $b(t)$ must evolve according to

$$
\frac{d}{d t} a^{2}\left(1+\frac{b^{2}}{n}\right)=\frac{Q}{\pi}, \quad a^{2} b=\text { constant }
$$


exactly as we found in $\S 3.2$ above.

It is clear from (3.14) that for ZST Stokes flow the singularities of the Schwarz function are fixed in the $\zeta$-plane (within the unit disc), rather than in $\Omega(t)$ in the $z$-plane, a fact which has profound implications for solutions. The example above illustrates how it is both awkward and unnecessary to bring the $z$-plane into the discussion when solving a problem for a given mapping function. For both Hele-Shaw and Stokes flow problems, driving singularities of the flow correspond to singularities of $g(z, t)$. For the Hele-Shaw problem, the singularities of $g(z, t)$ are fixed in the physical plane, by (2.9), and thus naturally correspond to fixed driving singularities. In Stokes flow however, only for flows with one driving singularity can we hope to keep it fixed in the physical plane. Multiple driving singularities must be determined in the $\zeta$-plane and thus in the physical plane they generally move in a way which cannot be prescribed there.

\subsection{The Baiocchi transformation}

Given the comments of $\S 2.4$, we may again define the function $u$ by the relation (2.14), and try to relate it to some physical quantity of interest in the problem. In this section we consider only the ZST problem, so we set $\gamma=0$ in (3.8). We deal with the NZST problem in $\S 4.5$.

As mentioned in $\S 2.3$, the Schwarz function is related to the conformal mapping from the unit disc by $g(z, t)=\bar{f}(1 / \zeta, t)$. Differentiating the definition (2.14) with respect to $\zeta$, it follows that

$$
\frac{\partial u}{\partial \zeta}=\frac{f^{\prime}(\zeta, t)}{4}(\overline{f(\zeta, t)}-\bar{f}(1 / \zeta, t))
$$

(we still write $u$ although the dependence is on $\zeta$ not $z$ ). Differentiating again with respect to $t$, and using (3.8) with $\gamma=0$,

$$
\frac{\partial^{2} u}{\partial t \partial \zeta}=\frac{1}{4} \frac{\partial}{\partial t}\left(f^{\prime}(\zeta, t) \overline{f(\zeta, t)}\right)+\frac{1}{2} \mathcal{X}^{\prime}(\zeta, t) .
$$

On the other hand, transferring (3.4) to the $\zeta$-plane,

$$
-2 \mathcal{A}(\zeta, t)=\overline{f(\zeta, t)} \Phi(\zeta, t)+\mathcal{X}(\zeta, t)+f(\zeta, t) \overline{\Phi(\zeta, t)}+\overline{\mathcal{X}(\zeta, t)} ;
$$

differentiating with respect to $\zeta$ and using (3.9) with $\gamma=0$ then yields

$$
-4 \frac{\partial \mathcal{A}(\zeta, t)}{\partial \zeta}=\overline{f(\zeta, t)} f_{t}^{\prime}(\zeta, t)+2 \mathcal{X}^{\prime}(\zeta, t)+f^{\prime}(\zeta, t) \overline{f_{t}(\zeta, t)} \equiv 4 \frac{\partial^{2} u}{\partial t \partial \zeta} .
$$

A final integration with respect to $\zeta$, using (3.3) and (2.16), gives

$$
\frac{\partial u}{\partial t}=-\mathcal{A}(\zeta, t)
$$

It should be emphasised that the derivative in this equation is with $\zeta$ fixed.

So here too we have a simple relationship between the Baiocchi variable $u$ (which again satisfies the system (2.15), (2.16)), and a physical quantity; however, here the relation holds in the $\zeta$-plane. The singularities of $u$, which of course correspond to those of the Schwarz function, are fixed in the $\zeta$-plane but move around in the physical plane. When 
formulated in terms of the Baiocchi variable $u$, the Stokes and Hele-Shaw problems thus differ only with regard to singularity motion, and the observations of this section go some way towards explaining the fact that solutions of the same functional form (but with different time dependence) exist for both Hele-Shaw and Stokes ZST flows with one singularity. In both cases the Baiocchi variable satisfies $\nabla^{2} u=1$ in the $z$-plane away from singularities; the key is to relate the Baiocchi variable to a physical quantity. When there is only one singularity, the form of the mappings for the two problems must be the same; singularity matching gives the time-dependence.

One well-known benefit of the Baiocchi-transformed version of the Hele-Shaw problem $((2.15)-(2.16)$ with specified singularities) is that time appears only as a parameter. In consequence, it is not necessary to know the solution at earlier times in order to find it at time $t$, and apart from calculating the time integrals of the driving singularities the problem need no longer be viewed as an evolutionary one (the Baiocchi transform thus has clear advantages for numerical, as well as analytical, approaches). A similar statement can now be given for the ZST Stokes problem. Writing $\nabla_{\zeta}$ for the gradient in the $\zeta$-plane, we have

$$
\begin{gathered}
\nabla_{\zeta}^{2} u=\left|\nabla_{\zeta}(\Re f(\zeta, t))\right|^{2}, \quad \nabla_{\zeta}^{2} \Re f(\zeta, t)=0 \quad \text { for }|\zeta|<1, \\
u=\frac{\partial u}{\partial n}=0 \quad \text { on }|\zeta|=1,
\end{gathered}
$$

with singularities specified in the $\zeta$-plane. Given $f(0, t)=0,(3.16)$ and (3.17) determine $f$ only up to a multiplicative factor $e^{i \Theta(t)}$, where $\Theta(t)$ is an arbitrary real function. This is to be expected in view of the invariance of Stokes flow under rigid body rotations. Time appears in (3.16), (3.17) only as a parameter; the nonlinearity in (2.15), (2.16) resides in the unknown free boundary location, while in (3.16), (3.17) it appears in the Poisson equation for $u$.

\subsection{Conserved quantities (Stokes flow moments)}

Analogous to the moments $M_{k}(t)$ defined for Hele-Shaw flow in $\S 2.5$, we may define quantities $\mathcal{M}_{k}(t)$ for the Stokes flow problem via

$$
\mathcal{M}_{k}(t)=\iint_{\Omega(t)} \zeta^{k}(z, t) d x d y
$$

for integers $k \geq 0$. For flow driven by a single point source (sink) of strength $Q>0$ $(Q<0)$ at the origin, it is straightforward to find the evolution equations for $\mathcal{M}_{k}(t)$, from (3.8) (see [10]; the discussion there parallels [8], and similar results also arise in $[48])$. The case $\gamma>0$ leads to a difficult system of nonlinear differential equations, but the ZST problem is simple, leading to an infinite system of conserved quantities exactly as for Hele-Shaw:

$$
\frac{d \mathcal{M}_{k}(t)}{d t}=Q \delta_{0 k}
$$

Note however that the result here relies on having $\zeta^{k}$ as the integrand, rather than $z^{k}$ in (2.18). In other words, the formulation is essentially in the $\zeta$-plane, and indeed, we could 
define

$$
\mathcal{M}_{k}(t)=\frac{1}{2 i} \oint_{|\zeta|=1} \zeta^{k} f^{\prime}(\zeta, t) \bar{f}(1 / \zeta, t) d \zeta,
$$

which can also be obtained by using Green's theorem on the definition (3.18). By contrast, the Hele-Shaw result was obtained quite independently of any considerations of the $\zeta$ plane.

As for Hele-Shaw, this result is also easily obtained via the Baiocchi transform. For the single point source problem, the Airy stress function $\mathcal{A}$ has the local behaviour $\mathcal{A} \sim-(Q /(2 \pi)) \log r$ as $r \rightarrow 0$, hence using the relation (3.15), the Baiocchi variable $u$ has the local behaviour in the $\zeta$-plane

$$
u=-\frac{Q t}{2 \pi} \log |\zeta|+\{\text { time-independent }\}+o(1) \quad \text { as }|\zeta| \rightarrow 0,
$$

and consequently

$$
\nabla^{2} u=1-\frac{Q t}{2 \pi} \delta(x) \delta(y) .
$$

Applying Green's theorem and using the boundary conditions (2.16),

$$
\iint_{\Omega(t)}\left(\zeta^{k} \nabla^{2} u-u \nabla^{2} \zeta^{k}\right) d x d y=\iint_{\Omega(t)} \zeta^{k} d x d y=Q t \delta_{0 k}+\{\text { time-independent }\}
$$

whence follows (3.19). Note that if there are two or more driving singularities, their positions have to be fixed in the $\zeta$-plane for the problem to be tractable, and we must apply Green's theorem there to derive equations for the moments. With just one singularity, we can perform the calculation in the $z$-plane because $f(0, t)=0$.

For completeness we give the NZST equations also. The mass conservation equation for $\mathcal{M}_{0}(t)$ is unchanged, while for $k \geq 1$ we have

$$
\frac{d \mathcal{M}_{k}(t)}{d t}=-\frac{k \gamma}{2 \mu} \sum_{r=0}^{\infty} \frac{G_{+}^{(r)}(0, t)}{r !} \mathcal{M}_{k+r}(t) .
$$

For the polynomial map of $\S 3.2$ the only nonzero moments are $\mathcal{M}_{0}$ and $\mathcal{M}_{n-1}$, with

$$
\mathcal{M}_{0}(t)=\pi a^{2}(t)\left(1+\frac{b^{2}(t)}{n}\right), \quad \mathcal{M}_{n-1}(t)=-\frac{\pi a^{2}(t) b(t)}{n},
$$

and equations (3.19) or (3.22) can be used to derive the solution. The moments provide a compact formulation for problems with a polynomial mapping function, but for a general rational mapping function they lead to an infinite system of coupled equations, which is an unnecessary complication (indeed, the question of whether the moments completely specify the motion in such cases is unclear, although it seems likely). However, as noted in $[\mathbf{1 0}]$, the quantity $\zeta^{k}$ in (3.20) can be replaced by an arbitrary function of $\zeta$, and a procedure for constructing conserved quantities of this type for rational maps is described in $[8]$.

There is again a relationship between these moments and the Schwarz function of the free boundary. Comparing the representation (3.20) with (3.13) reveals the $\mathcal{M}_{k}(t)$ to be the coefficients of the principal part of the Laurent expansion of $H^{\prime}(\zeta, t)$ about $\zeta=0$. This is very similar to the Hele-Shaw result (2.19), though again with the important difference that we are forced to work in the $\zeta$-plane. 


\subsection{A quadrupole in a circle}

In $§ 2.6$ we presented a deductive procedure for finding the form of the mapping function for a particular initial-value ZST Hele-Shaw problem (see also [38]). In this section we illustrate the analogous procedure for the Stokes flow problem under the assumption that $\phi(0, t)=0$. As mentioned in $\S 3$, this assumption is justified for problems with sufficient symmetry, so we choose such an example: a quadrupole singularity, situated at the centre of an initially-circular fluid domain. The methods we use are easily extended to other (more complicated) examples. We consider only the ZST problem, conjecturing (by singularity-matching arguments) that there is an NZST solution $f(\zeta, t)$ to $(3.8)$ and (3.9) with the same functional form (but with different time-dependence of the parameters) if and only if a ZST solution exists.

The argument parallels that of $\S 2.6$. We decompose the functions $\mathcal{X}(\zeta, t)$ and $H(\zeta, t)$ (introduced at the start of §3.3) into their analytic and singular parts within the unit disc. In terms of the Stokes flow moments $\mathcal{M}_{k}(t)$, recalling the comments of $\S 3.3$ and again using the subscript ' $e$ ' to denote the part of an analytic function that is singular inside $|\zeta|=1$ and regular in its exterior, we have

$$
H_{e}^{\prime}(\zeta, t)=\frac{1}{\pi} \sum_{0}^{\infty} \frac{\mathcal{M}_{k}(t)}{\zeta^{k+1}} .
$$

Then (3.14) gives

$$
\frac{\partial H_{e}(\zeta, t)}{\partial t}=-2 \mathcal{X}_{e}(\zeta, t)
$$

where $\mathcal{X}_{e}(\zeta, t)$ is known precisely once the driving mechanism is prescribed. For a quadrupole singularity of strength $M$ at the origin, the Goursat function $\chi(z, t)$ has the local behaviour

$$
\chi(z, t)=\frac{M}{z^{2}}+O(1)
$$

being regular elsewhere, while $\phi(z, t)$ is regular everywhere. It follows that

$$
\mathcal{X}_{e}(\zeta, t)=\frac{M}{\zeta^{2}\left(f^{\prime}(0, t)\right)^{2}}-\frac{M f^{\prime \prime}(0, t)}{\zeta\left(f^{\prime}(0, t)\right)^{3}},
$$

so that integrating with respect to time in (3.24),

$$
H_{e}(\zeta, t)=H_{e}(\zeta, 0)-\frac{2 M \theta_{1}(t)}{\zeta^{2}}+\frac{2 M \theta_{2}(t)}{\zeta},
$$

where

$$
\theta_{1}(t)=\int_{0}^{t} \frac{d t^{\prime}}{\left(f^{\prime}\left(0, t^{\prime}\right)\right)^{2}} \quad \text { and } \quad \theta_{2}(t)=\int_{0}^{t} \frac{f^{\prime \prime}\left(0, t^{\prime}\right) d t^{\prime}}{\left(f^{\prime}\left(0, t^{\prime}\right)\right)^{3}}
$$

are unknown functions of time (the conformal map being as yet unspecified). $H_{e}(\zeta, 0)$ is determined by the initial geometry, which here is a circle of radius $r$ centred on the origin: the Schwarz function of $\partial \Omega(0)$ is $g(z, 0)=r^{2} / z$, giving

$$
h_{e}(z, 0)=r^{2} \log z, \quad H_{e}(\zeta, 0)=r^{2} \log \zeta .
$$


Hence

$$
H_{e}^{\prime}(\zeta, t)=\frac{r^{2}}{\zeta}-\frac{2 M \theta_{2}(t)}{\zeta^{2}}+\frac{4 M \theta_{1}(t)}{\zeta^{3}} \equiv\left[f^{\prime}(\zeta, t) \bar{f}(1 / \zeta, t)\right]_{e},
$$

where we used (3.13) in the last equality. Since $f^{\prime}(\zeta, t)$ is analytic within the unit disc (3.26) gives the singularities of $\bar{f}(1 / \zeta, t)$ there, namely a triple pole at the origin (and no other singularities), so $f(\zeta, t)$ must have the form

$$
f(\zeta, t)=a(t) \zeta+b(t) \zeta^{2}+c(t) \zeta^{3}
$$

exactly as for the Hele-Shaw example. Again, since $\Omega(t)$ is symmetric about both coordinate axes, $b(t) \equiv 0$. We solve for $a(t)$ and $c(t)$ by matching the singularity at the origin in (3.8) (with $\gamma=0$ ). Near $\zeta=0$,

$$
f^{\prime}(\zeta, t) \bar{f}(1 / \zeta, t)=\frac{a c}{\zeta^{3}}+\frac{1}{\zeta}\left(a^{2}+3 c^{2}\right)+O(1)
$$

and using (3.25),

$$
\mathcal{X}(\zeta, t)=\frac{M}{a^{2} \zeta^{2}}+O(1)
$$

Hence we find

$$
\frac{d}{d t}\left(a^{2}+3 c^{2}\right)=0, \quad \frac{d}{d t}(a c)=\frac{4 M}{a^{2}}
$$

note that these equations differ from (2.22) and (2.23). We fix the constant fluid area equal to $\pi$ by taking $a(0)=1$ and $c(0)=0$. Making the substitutions $a=\sin \theta(t)$, $c=\cos \theta(t) / \sqrt{3}$, where $\theta(0)=\pi / 2$,

$$
\frac{d \theta}{d t}(1-2 \cos 2 \theta+\cos 4 \theta)=-16 M \sqrt{3},
$$

and hence

$$
\theta-\sin 2 \theta+\frac{1}{4} \sin 4 \theta=-16 M t \sqrt{3}+\frac{\pi}{2} .
$$

The evolution is illustrated in figure 3 . The domain evolves smoothly from its initial circular form until time $t^{*}=(\pi+4) /(64 M \sqrt{3})$, at which point $\theta=\pi / 4, d \theta / d t=-\infty$ and the solution blows up with the simultaneous formation of two cusps (as did the Hele-Shaw solution). We discuss blow-up further below.

\subsection{Linear stability and blow-up}

As the examples of $\S 3.2$ and $\S 3.6$ show, it is possible to have finite-time blow-up of ZST Stokes flow solutions, with a cusp forming in the moving boundary. Indeed, given the remarks in $\S 3.4$, it is likely that we can find Stokes blow-up corresponding to any known form of Hele-Shaw blow-up generated by a single driving singularity, by using the same conformal map. It should be noted, though, that the different time-dependence in the two problems may give rise to different phenomena. For example, in Hele-Shaw flow, continuation is possible beyond cusps of 5/2-power type, which does not always appear to be the case for Stokes flow. Furthermore, the presence of surface tension in Stokes flow may postpone the advent of singularities until the final moment of the flow. For 


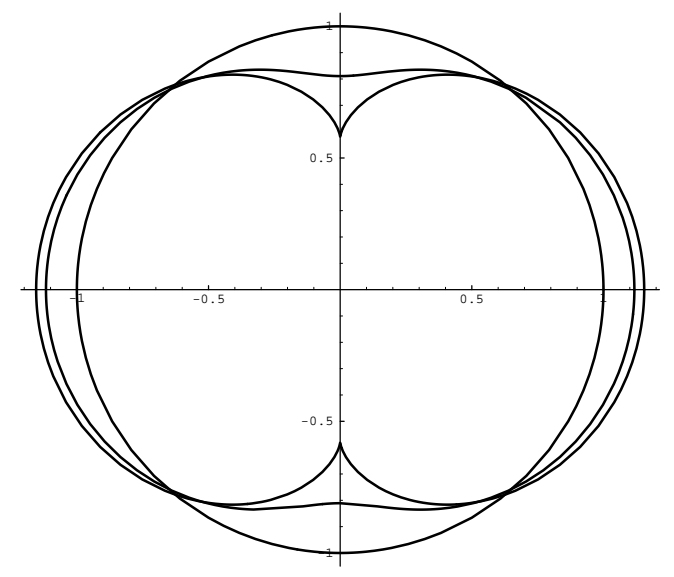

FIGURE 3. Evolution of an initially circular region containing a quadrupole at the origin, up to the time of cusp formation.

instance, in the example of $\S 3.2$, when $\gamma>0$, cusps form on the moving boundary at the same time as the fluid area reaches zero. Note, too, that cusps can form in NZST Stokes flows evolving under the action of surface tension alone, i.e. with no external driving singularities. Among the remarkable solutions of [44] is one which starts with a symmetric initial configuration of four large circles touching one smaller central circle, and evolves to a single circle as $t \rightarrow \infty$. On the way, the moving boundary, which is otherwise smooth (analytic) everywhere, spontaneously develops four (because of symmetry) cusps of 5/2-power type. This cusp formation might be termed 'geometrically necessary', as it occurs on the borderline between solutions whose initial conditions are such that the fluid domain remains simply-connected for all time, and those whose initial conditions lead to overlapping at a finite time. This purely topological argument suggests that such solutions are possible for NZST Hele-Shaw flows as well, and indeed for any system that can change its topology by overlapping.

Unlike the Hele-Shaw problem though, we cannot classify Stokes flow problems as 'advancing' or 'receding' free boundary cases, because there is no maximum principle for solutions of the biharmonic equation. Moreover, for Stokes flow, the invariance under rigid-body motion means that it makes little sense to talk about the local linear stability of advancing/retreating viscous boundaries. However, we are able to analyse the stability of nearly-circular blobs and bubbles in ZST Stokes flow very simply, by exploiting the family of exact solutions given in $§ 3.2$. 
In the case $b(t)=\epsilon(t) \ll 1$, the mapping function (2.5) has free boundary

$$
|z|=a\left(1-\frac{\epsilon}{n} \cos (n-1) \theta+O\left(\epsilon^{2}\right)\right)
$$

which is just a sinusoidal perturbation to an expanding or contracting circle, as would arise in a linear stability analysis. From the results of $\S 3.2$, the (ZST) evolution of $a(t)$ and $\epsilon(t)$ is given by

$$
a^{2} \epsilon=k, \quad \frac{d S}{d t}=\frac{d}{d t}\left(\pi a^{2}\left(1+\frac{\epsilon^{2}}{n}\right)\right)=Q
$$

where $k$ is a positive constant and $S(t)$ denotes the area of the fluid domain. To lowest order these give the solution for $\epsilon(t)$ as

$$
\epsilon(t)=\epsilon(0) \frac{S(0)}{S(t)}
$$

Hence $\epsilon(t)$ grows in time for a point sink $(S(t)$ decreasing), which means an unstable situation, and decreases in time for a point source $(S(t)$ increasing), which means a stable situation. Thus for viscous blobs, we have the same situation as for Hele-Shaw, but it is important to note that the growth or decay here is algebraic in $t$, in contrast to the exponential growth/decay in time observed with the corresponding Hele-Shaw stability analysis (see for example [34]). Furthermore, until $S(t)$ is small (at which point the linearisation is no longer valid), the growth rate is independent of $n$, again in contrast with the Hele-Shaw case. The reason is that a planar interface is neutrally stable, as Stokes flow is indifferent to rigid body motion. The instability for Stokes flow is as much induced by the geometry as by any more intrinsic properties of the free boundary, and is correspondingly less pronounced.

To analyse nearly-circular bubbles we exploit a similar solution (due to Tanveer \& Vasconcelos [48]), with mapping function

$$
z=f(\zeta, t)=a\left(\frac{1}{\zeta}-\frac{\epsilon}{n} \zeta^{n}\right)
$$

again $a(t)>0$ and $0<\epsilon(t) \ll 1 .^{7}$ The resulting free boundary has

$$
|z|=a\left(1-\frac{\epsilon}{n} \cos (n+1) \theta+O\left(\epsilon^{2}\right)\right)
$$

and the ZST equations for the parameters are

$$
a^{2} \epsilon=k, \quad \frac{d B}{d t}=\frac{d}{d t}\left(\pi a^{2}\left(1-\frac{\epsilon^{2}}{n}\right)\right)=Q,
$$

where now $B(t)$ denotes the bubble area, so $Q>0$ for a sink at infinity (a growing bubble), and $Q<0$ for a shrinking bubble. To lowest order these have solution

$$
\epsilon(t)=\epsilon(0) \frac{B(0)}{B(t)}
$$

so an expanding bubble is stable ( $\epsilon$ decreasing), while a shrinking bubble is unstable,

7 Note that $f(0, t)=\infty$, since the driving mechanism is at infinity in this problem. We have not discussed such maps, but the theory is a straightforward extension of that with $f(0, t)=0$. 
the decay/growth of perturbations again being algebraic in time. This result is in direct contrast to the corresponding Hele-Shaw result, in which perturbations to an expanding/shrinking bubble initially exhibit exponential growth/decay (again, see [34]).

\subsection{Summary}

In $\S \S 2$ and 3 we have developed the complex variable theory for the two problems, along parallel lines. We now briefly summarise the main results, for a direct comparison.

- Both problems are quasistatic (time-dependence entering only via the kinematic boundary conditions), and governed by elliptic partial differential equations: the pressure field in Hele-Shaw is harmonic, and both stream- and stress-functions for (two-dimensional) Stokes flow are biharmonic.

- As a consequence, both problems lend themselves to complex variable (conformal mapping) methods of solution.

- Many explicit solutions involving rational and log-rational conformal maps can be written down for both problems.

- The NZST Stokes flow problem is much more tractable analytically than the NZST Hele-Shaw problem, with many exact time-dependent solutions (both with and without driving singularities) existing in the literature.

- For both problems, a global equation can be derived for the Schwarz function of the free boundary (equations (2.9) and (3.14)). The time-varying singularities of the Schwarz function are thus seen to correspond to driving singularities of the problems; however, this correspondence is in the physical plane for Hele-Shaw flow and in the $\zeta$-plane for ZST Stokes flow. This forces us to consider moving singularities in many Stokes flow problems. In particular, we note the following:

- If there is just one driving singularity at a finite point, momentum is not in general conserved unless the singularity moves in a specific manner. Exceptions include symmetric domains for which momentum is automatically conserved.

- If there are two or more singularities, for existing complex variable methods to apply they must be allowed to move even if momentum conservation is automatic. This also applies to domains extending to infinity, with a single singularity at a finite point, as the conditions at infinity induce a singularity there.

- Baiocchi transformations may be defined for both problems as outlined in $\S \S 2.4,3.4$. For Hele-Shaw flow the Baiocchi variable is the time integral of the pressure in the $z$-plane; for ZST Stokes flow it is the time integral of the Airy stress function in the $\zeta$-plane. An appropriate time integral of the Airy stress function also performs this rôle in the NZST Stokes problem: see $\S 4.5$.

- Infinite sets of conserved quantities exist for the ZST problems; again, for Hele-Shaw these are defined most naturally in the physical plane, and in the $\zeta$-plane for ZST Stokes flow.

- The Hele-Shaw model with retreating free boundary (the 'suction problem') is always unstable (in fact, ill-posed) leading to finite-time blow-up (often via cusp formation). In particular, expanding bubbles and contracting blobs are unstable, while the converse 
situations are stable. For Stokes flow, stability is less clear-cut; however the analysis of nearly-circular geometries gave the contrasting results that contracting blobs and bubbles are unstable (the converse situations being unstable). Moreover, the mode of blow-up differs for the two problems, Hele-Shaw being exponential in time, while Stokes flow is only algebraic.

\section{Stokes flow: further developments}

\subsection{Preamble}

In this section we expand upon the sketch of $\S 3$. We have seen that this theory is often unsatisfactory for describing singularity-driven flows; here we extend it to allow a general (one-singularity) flow to be dealt with in a 'physically realistic' manner. We also sketch several extensions of the theory, to account for surface tension in the Baiocchi framework, and to flows with gravity.

We begin by demonstrating how the difficulties noted so far are linked to the assumption of $\S 3$ that the Goursat function $\phi(z, t)$ may be assumed to vanish at the origin, as discussed in $\S 3$. Suppose we have a Stokes flow driven solely by surface tension, and no driving singularity. The invariance under rigid-body motion means that there exists a family of possible solutions, which can be generated from any one solution by adding on arbitrary translations and/or rotations to the velocity field. Suppose now that $(\phi, \chi)$ is the unique solution having zero net momentum. Clearly $\phi$ does not in general vanish at the origin, so assume $\phi(0, t)=A(t)$ (arbitrary and complex) and consider the second Goursat pair

$$
\widetilde{\phi}=\phi-A-B z, \quad \widetilde{\chi}=\chi+C z .
$$

It is easily checked that if the first pair is a solution to the free boundary problem then so is the second, provided $C=\bar{A}$ and $B=i \lambda$ for some $\lambda \in \mathbb{R}$ (the force balance condition (3.7) is the same for each, and by (3.5) the pressure fields are identical). However, by (3.6) the corresponding velocity fields then differ according to

$$
\tilde{u_{1}}+i \tilde{u_{2}}=u_{1}+i u_{1}-2(A+i \lambda z) .
$$

The second pair has the feature $\widetilde{\phi}(0, t)=0$, which will be seen to simplify the solution procedure considerably (in particular, a polynomial mapping function will yield a solution if and only if this condition holds), but has nonzero net linear and angular momentum, as (4.1) shows.

If there is no driving singularity, this is of no consequence: we may solve for the simpler case $\phi(0, t)=0$ and subtract off the appropriate rigid body motion a posteriori if necessary. However, if we wish to solve for a fixed driving mechanism such as a source or a sink, then doing this gives rise to a solution which is contrived, since it must have the singularity moving in a particular manner within the fluid. Assuming $\phi(0, t)=0$ is not always satisfactory therefore, and we shall now consider the more general situation when it is finite and nonzero. 


\subsection{Details of the theory for Stokes flow}

To extend the theory as indicated above, we go back and fill in some of the details of the complex variable framework, following a slightly different approach to that of $[\mathbf{4 1}]$. From now on we allow $\phi(0, t)$ to be nonzero; however in most of the following we assume it to be bounded, which limits the kind of driving singularities we can deal with. The basic ideas we present may be extended to deal with singular behaviour in $\phi$ at the origin, for example due to a multipole, but the algebra is worse.

Using $u_{t}(z), u_{n}(z)$ to denote the tangential and normal components of the fluid velocity $^{8}$ (both real), at a point $z$ on $\partial \Omega(t)$, we have

$$
\left.\left(u_{1}+i u_{2}\right)\right|_{\partial \Omega(t)}=\left(u_{t}-i u_{n}\right) \frac{\partial z}{\partial s} .
$$

Hence from (3.6) we see that

$$
\phi(z, t)-z \overline{\phi^{\prime}(z, t)}-\overline{\chi^{\prime}(z, t)}=\left(u_{t}(z, t)-i u_{n}(z, t)\right) \frac{\partial z}{\partial s} \quad \text { on } \partial \Omega(t),
$$

and this holds together with the force balance condition (3.7). The left-hand side here is easily rewritten in terms of $\zeta$; for the right-hand side we note that, with $\zeta=e^{i \theta}$,

$$
\frac{\partial z}{\partial s}=f^{\prime}\left(e^{i \theta}, t\right) \frac{d\left(e^{i \theta}\right)}{d s}=i \zeta f^{\prime}(\zeta, t) \frac{d \theta}{d s}=i \zeta \frac{f^{\prime}(\zeta, t)}{\left|f^{\prime}(\zeta, t)\right|} .
$$

In the third equality here we used the facts that $|\partial z / \partial s|=1$, and that $d \theta / d s$ is real and positive for the anticlockwise tangent. The boundary conditions (4.2) and (3.7) then become

$$
\begin{aligned}
& \Phi(\zeta, t)-f(\zeta, t) \frac{\overline{\Phi^{\prime}(\zeta, t)}}{\overline{f^{\prime}(\zeta, t)}}-\frac{\overline{\mathcal{X}^{\prime}(\zeta, t)}}{\overline{f^{\prime}(\zeta, t)}}=i \zeta\left(U_{t}(\zeta, t)-i U_{n}(\zeta, t)\right) \frac{f^{\prime}(\zeta, t)}{\left|f^{\prime}(\zeta, t)\right|}, \\
& \Phi(\zeta, t)+f(\zeta, t) \frac{\overline{\Phi^{\prime}(\zeta, t)}}{\overline{f^{\prime}(\zeta, t)}}+\frac{\overline{\mathcal{X}^{\prime}(\zeta, t)}}{\overline{f^{\prime}(\zeta, t)}}=-\frac{\gamma}{2 \mu} \zeta \frac{f^{\prime}(\zeta, t)}{\left|f^{\prime}(\zeta, t)\right|}
\end{aligned}
$$

both holding on $|\zeta|=1$. Here $U_{t}(\zeta, t)$ and $U_{n}(\zeta, t)$ denote the tangential and normal components of the fluid velocity in the $\zeta$-plane. Adding (4.3) and (4.4) gives

$$
\frac{2 \Phi(\zeta, t)}{\zeta f^{\prime}(\zeta, t)}=\frac{1}{\left|f^{\prime}(\zeta, t)\right|}\left(U_{n}(\zeta, t)+i U_{t}(\zeta, t)-\frac{\gamma}{2 \mu}\right) \quad \text { on }|\zeta|=1 .
$$

In addition, elementary consideration of a boundary point $z(t)=f\left(e^{i \theta(t)}, t\right)$ shows

$$
\left.\left(u_{1}+i u_{2}\right)\right|_{\partial \Omega(t)}=i \zeta f^{\prime}(\zeta, t) \frac{d \theta}{d t}+\frac{\partial f}{\partial t}(\zeta, t),
$$

while equations (3.6) and (3.7) combine to give

$$
\left.\left(u_{1}+i u_{2}\right)\right|_{\partial \Omega(t)}=2 \phi(z, t)-\frac{i \gamma}{2 \mu} \frac{\partial z}{\partial s} .
$$

Equating these two expressions we find

$$
\frac{1}{\zeta f^{\prime}(\zeta, t)}\left(2 \Phi(\zeta, t)-\frac{\partial f}{\partial t}(\zeta, t)\right)+\frac{\gamma}{2 \mu} \frac{1}{\left|f^{\prime}(\zeta, t)\right|}=i \frac{d \theta}{d t}, \quad \text { on }|\zeta|=1 .
$$

8 These are measured so that $u_{n}>0$ if the motion is along the outward normal, and $u_{t}>0$ if the velocity is along the anticlockwise tangent vector. 
Comparison of (4.5) and (4.7) gives

$$
\begin{aligned}
\frac{U_{n}}{\left|f^{\prime}(\zeta, t)\right|} & =\Re\left(\frac{f_{t}(\zeta, t)}{\zeta f^{\prime}(\zeta, t)}\right), \\
\frac{U_{t}}{\left|f^{\prime}(\zeta, t)\right|} & =\Im\left(\frac{f_{t}(\zeta, t)}{\zeta f^{\prime}(\zeta, t)}\right)+\frac{d \theta}{d t} .
\end{aligned}
$$

Adding equations (4.2) and (3.7) yields

$$
2 \Im\left[\phi(z, t) \frac{d \bar{z}}{d s}\right]=\frac{\gamma}{2 \mu}-u_{n},
$$

while (3.7) alone gives

$$
\phi(z, t) \frac{\partial \bar{z}}{\partial s}+z \overline{\phi^{\prime}(z, t)} \frac{\partial \bar{z}}{\partial s}+\overline{\chi^{\prime}(z, t)} \frac{\partial \bar{z}}{\partial s}=\frac{i \gamma}{2 \mu} .
$$

Eliminating $\gamma /(2 \mu)$ between the last two equations gives

$$
\frac{\partial}{\partial s}(\bar{z} \phi(z, t)+\chi(z, t))=-i u_{n} .
$$

For the steady problem $u_{n} \equiv 0$ and this is just the 'streamline condition', that both the streamfunction and the Airy stress function are constant on the free boundary. For the time-dependent problem we recast the equation in terms of $\zeta$ using (4.8) to find, after some rearrangement, the condition

$$
\frac{\partial}{\partial \zeta}\left(\mathcal{X}(\zeta, t)+\bar{f}(1 / \zeta, t)\left[\Phi(\zeta, t)-\frac{1}{2} f_{t}(\zeta, t)\right]\right)+\frac{1}{2} \frac{\partial}{\partial t}\left(f^{\prime}(\zeta, t) \bar{f}(1 / \zeta, t)\right)=0,
$$

holding on $|\zeta|=1$, and elsewhere by analytic continuation. Note that equation (4.10) assumes nothing about the behaviour of $\phi(z, t)$ at the origin, nor does it contain the surface tension parameter $\gamma$; however, it does contain both of the unknown Goursat functions, which limits its application.

We need one more equation to close the problem; for this we return to (4.7) and observe that the real part of the left-hand side must vanish on $|\zeta|=1$. Analytic continuation is complicated by the square-root branch point in $\left|f^{\prime}(\zeta, t)\right|$, but (following [41]) this is dealt with by introducing functions $\mathcal{F}_{+}(\zeta, t), \mathcal{F}_{-}(\zeta, t)$, analytic on $|\zeta|<1,|\zeta|>1$ respectively, such that

$$
\frac{1}{\left(f^{\prime}(\zeta, t) \bar{f}^{\prime}(1 / \zeta, t)\right)^{1 / 2}}=\mathcal{F}_{+}(\zeta, t)-\mathcal{F}_{-}(\zeta, t)
$$

These functions are unique if we also insist that $\mathcal{F}_{-}(\zeta, t)$ vanish as $|\zeta| \rightarrow \infty$, and have the explicit representation ( $c f .(3.10))$

$$
\mathcal{F}_{ \pm}(\zeta, t)=\frac{1}{2 \pi i} \oint_{|\tau|=1} \frac{1}{\left|f^{\prime}(\tau, t)\right|} \frac{d \tau}{(\tau-\zeta)}
$$

for $|\zeta|<1$ and $|\zeta|>1$ respectively. Condition (4.7) can thus be rewritten as

$$
\Re\left(\frac{1}{\zeta f^{\prime}(\zeta, t)}\left[2 \Phi(\zeta, t)-\frac{\partial f}{\partial t}(\zeta, t)\right]+\frac{\gamma}{\mu} \mathcal{F}_{+}(\zeta, t)\right)=\frac{\gamma}{2 \mu} \mathcal{F}_{+}(0, t), \quad \text { on }|\zeta|=1 .
$$

This boundary condition may be analytically continued away from the unit circle, but this 
continuation depends on the behaviour of the function $\Phi$ at the origin. When $\Phi(0, t)=0$ it is easily checked that the continuation is given by equation $(3.9)$, noting that $G_{+}(\zeta, t)=$ $2 \mathcal{F}_{+}(\zeta, t)-\mathcal{F}_{+}(0, t)$; when $\Phi(0, t)=A(t)$ (finite and nonzero) the continuation is

$$
2 \Phi(\zeta, t)-\frac{\partial f}{\partial t}(\zeta, t)+\frac{\gamma}{2 \mu} G_{+}(\zeta, t) \zeta f^{\prime}(\zeta, t)=2 \frac{f^{\prime}(\zeta, t)}{f^{\prime}(0, t)}\left(A(t)-\overline{A(t)} \zeta^{2}\right) .
$$

In particular, we note that when $A \neq 0$, polynomial maps no longer work; if $f(\zeta, t)$ is a polynomial of degree $n$ then the term in $A$ has a pole of order $(n+1)$, and there is no balancing term. Expressions for cases of singular behaviour of $\Phi(\zeta, t)$ at the origin (e.g. poles) may be written down [9], but they are more complicated, and the above is the only generalisation we consider.

\subsection{A hyperbolic partial differential equation for the Schwarz function}

\subsubsection{Formulation}

We saw in $§ 3.6$ how, by solving a simple differential equation (3.24) for the function $H_{e}(\zeta, t)$ (whose derivative is the part of the Schwarz function that is singular within $|\zeta|=1$ ), we are able to deduce the form of the mapping function for a particular geometry and driving mechanism. When we drop the assumption $\phi(0, t)=0$ equation (3.24) no longer holds, and in this section we find and solve its replacement.

Setting $A(t)=P(t) e^{i \beta(t)}$ for real $P(t), \beta(t)$, we define

$$
\check{\zeta}=\zeta e^{-i \beta(t)},
$$

and write $\check{f}(\check{\zeta}, t)=f(\zeta, t)$ (and similarly for other functions of $\zeta$ ). Equations (4.10) and (4.11) then combine to give

$$
\begin{aligned}
& \frac{\partial}{\partial t}\left(\check{f}^{\prime}(\check{\zeta}, t) \overline{\check{f}}(1 / \check{\zeta}, t)\right)-i \frac{d \beta}{d t} \check{f}^{\prime}(\check{\zeta}, t) \bar{f}(1 / \check{\zeta}, t)+2 \check{\mathcal{X}}^{\prime}(\check{\zeta}, t) \\
& \quad+\frac{2 A(t)}{\check{f}^{\prime}(0, t)} \frac{\partial}{\partial \check{\zeta}}\left(\left(1-\check{\zeta}^{2}\right) \check{f}^{\prime}(\check{\zeta}, t) \overline{\check{f}}(1 / \check{\zeta}, t)\right)=\frac{\gamma}{2 \mu} \frac{\partial}{\partial \check{\zeta}}\left(\check{\zeta} \check{f}^{\prime}(\check{\zeta}, t) \overline{\check{f}}(1 / \check{\zeta}, t) \check{G}+(\check{\zeta}, t)\right)
\end{aligned}
$$

(here the prime denotes $\partial / \partial \check{\zeta}$ ), which reduces to (3.8) when $A(t)=0=\beta(t)$.

We now assume zero surface tension, for simplicity. The relevant governing equations are then (4.11) and (4.12), with $\gamma=0$. From (3.13) we have

$$
H^{\prime}(\zeta, t)=f^{\prime}(\zeta, t) \bar{f}(1 / \zeta, t)=e^{-i \beta} \check{f}^{\prime}(\check{\zeta}, t) \bar{f}(1 / \check{\zeta}, t)=e^{-i \beta} \check{H}^{\prime}(\check{\zeta}, t)
$$

Equation (4.12) then becomes

$$
\frac{\partial}{\partial t}\left(e^{-i \beta} \check{H}^{\prime}(\check{\zeta}, t)\right)+2 e^{-i \beta} \check{\mathcal{X}}^{\prime}(\check{\zeta}, t)+\frac{2 A(t)}{\check{f}^{\prime}(0, t)} \frac{\partial}{\partial \check{\zeta}}\left(\left(1-\check{\zeta}^{2}\right) e^{-i \beta} \check{H}^{\prime}(\check{\zeta}, t)\right)=0,
$$

which may be integrated once with respect to $\check{\zeta}$ giving

$$
\frac{\partial\left(e^{-i \beta} \check{H}\right)}{\partial t}+\frac{2 A(t)}{\check{f}^{\prime}(0, t)}\left(1-\check{\zeta}^{2}\right) \frac{\partial\left(e^{-i \beta} \check{H}\right)}{\partial \check{\zeta}}+2 e^{-i \beta} \check{\mathcal{X}}(\check{\zeta}, t)=0,
$$

where the arbitrary function of time has been absorbed into $\check{\mathcal{X}}(\check{\zeta}, t)$ without loss of generality. This equation replaces (3.14). Note that the combination $A(t) / \check{f}^{\prime}(0, t)$ is real, being 
exactly $P(t) / f^{\prime}(0, t)$. Henceforth we drop the inverted hats on functions and variables, on the understanding that we are working with $e^{-i \beta} \check{H}, e^{-i \beta} \check{\mathcal{X}}$ and a real $A(t)$; they do not affect the analysis and are not needed in any case if $\phi(0, t)$ is real.

We want to take the singular part of this partial differential equation within the unit disc, since we know $\mathcal{X}_{e}(\zeta, t)$ precisely for a given driving mechanism. When doing this, we must remember to subtract off the regular terms arising from the term $-\left(2 A(t) \zeta^{2} / f^{\prime}(0, t)\right) \partial H_{e} / \partial \zeta$. The result, using (3.23), is

$$
\frac{\partial H_{e}}{\partial t}+\frac{2 A(t)}{f^{\prime}(0, t)}\left(1-\zeta^{2}\right) \frac{\partial H_{e}}{\partial \zeta}+2 \mathcal{X}_{e}(\zeta, t)=-\frac{2 A(t)}{\pi f^{\prime}(0, t)}\left(\mathcal{M}_{0}(t) \zeta+\mathcal{M}_{1}(t)\right),
$$

where $\mathcal{M}_{0}$ and $\mathcal{M}_{1}$ are, respectively, the zeroth and first moments as defined by (3.18). (This result is quite general; for a specific driving mechanism one or other of $\mathcal{M}_{0}$ and $\mathcal{M}_{1}$ may be constant.) Alternatively, defining the scaled time variable $\tau$ by

$$
\frac{d \tau}{d t}=\frac{2 A(t)}{f^{\prime}(0, t)}
$$

with $\tau(0)=0$,

$$
\frac{\partial H_{e}}{\partial \tau}+\left(1-\zeta^{2}\right) \frac{\partial H_{e}}{\partial \zeta}=-\frac{1}{\pi}\left(\mathcal{M}_{0}(\tau) \zeta+\mathcal{M}_{1}(\tau)\right)-\frac{f^{\prime}(0, \tau)}{A(\tau)} \mathcal{X}_{e}(\zeta, \tau) .
$$

(We abuse notation and use the same letters for the functions whether the dependence is on $t$ or $\tau$.) Defining the function

$$
\mathcal{H}(\zeta, \tau)=\sum_{k=1}^{\infty} \frac{\mathcal{M}_{k}(\tau)}{k \zeta^{k}}=\mathcal{M}_{0}(\tau) \log \zeta-\pi H_{e}(\zeta, \tau)
$$

(4.13) then becomes

$$
\frac{\partial \mathcal{H}}{\partial \tau}+\left(1-\zeta^{2}\right) \frac{\partial \mathcal{H}}{\partial \zeta}=\frac{\mathcal{M}_{0}}{\zeta}+\mathcal{M}_{1}+\frac{\pi f^{\prime}(0, \tau)}{A(\tau)} \hat{\mathcal{X}}_{e}(\zeta, \tau),
$$

where $\hat{\mathcal{X}}_{e}(\zeta, \tau):=\mathcal{X}_{e}(\zeta, \tau)+(Q / 2 \pi) \log \zeta$, so we have subtracted off any point source/sink behaviour. If, for example, we have a flow driven only by a point source/sink, then $\hat{\mathcal{X}}_{e}(\zeta, \tau) \equiv 0$ and the partial differential equation for $\mathcal{H}$ is just

$$
\frac{\partial \mathcal{H}}{\partial \tau}+\left(1-\zeta^{2}\right) \frac{\partial \mathcal{H}}{\partial \zeta}=\frac{\mathcal{M}_{0}(\tau)}{\zeta}+\mathcal{M}_{1}
$$

If on the other hand we have a dipole of strength $M$ at the origin (and no point sink) then $\hat{\mathcal{X}}_{e}(\zeta, \tau) \equiv \mathcal{X}_{e}(\zeta, \tau)=M /\left(f^{\prime}(0, \tau) \zeta\right)$. This is essentially the same as the case $\hat{\mathcal{X}}_{e}(\zeta, \tau) \equiv 0$ (only the coefficient of $1 / \zeta$ in (4.16) is modified), so we can find the general solution of the partial differential equation in this case too with no extra work. With no point sink or source, $\mathcal{M}_{0}$ is just a positive constant equal to the area of the fluid domain.

Defining $\hat{\mathcal{H}}=\mathcal{H}-\int^{\tau} \mathcal{M}_{1}\left(\tau^{\prime}\right) d \tau^{\prime}$, (4.16) becomes

$$
\frac{\partial \hat{\mathcal{H}}}{\partial \tau}+\left(1-\zeta^{2}\right) \frac{\partial \hat{\mathcal{H}}}{\partial \zeta}=\frac{\mathcal{M}_{0}(\tau)}{\zeta} .
$$

The characteristic projections in the $(\zeta, \tau)$-plane are

$$
\zeta=\tanh \left(\tau+\tanh ^{-1} \zeta_{0}\right)
$$


for some parameter $\zeta_{0}$. Equivalently, the combination

$$
\tanh ^{-1} \zeta_{0}=\tanh ^{-1} \zeta-\tau
$$

is constant along a characteristic. On characteristics,

$$
\frac{d \hat{\mathcal{H}}}{d \tau}=\frac{\mathcal{M}_{0}(\tau)}{\tanh \left(\tau+\tanh ^{-1} \zeta_{0}\right)}
$$

so the solution to $(4.16)$ is

$$
\mathcal{H}(\zeta, \tau)=\int_{0}^{\tau} \frac{\mathcal{M}_{0}\left(\tau^{\prime}\right) d \tau^{\prime}}{\tanh \left(\tau^{\prime}-\tau+\tanh ^{-1} \zeta\right)}+\mathcal{H}_{0}\left(\frac{\zeta-\tanh \tau}{1-\zeta \tanh \tau}\right)+\int_{0}^{\tau} \mathcal{M}_{1}\left(\tau^{\prime}\right) d \tau^{\prime}
$$

where $\mathcal{H}_{0}(\zeta)=\mathcal{H}(\zeta, 0)$ depends on the initial conditions.

\subsubsection{Stokes moments when $\phi(0, t) \neq 0$}

We are still able to recover the Stokes flow moments in the more complicated case $\phi(0, t)=A(t) \neq 0$. We can do this either by noting that by (3.23) the $\mathcal{M}_{k}$ are the Laurent coefficients of the function $H_{e}^{\prime}(\zeta, \tau)$, or more directly, by considering the system of ordinary differential equations satisfied by the $\mathcal{M}_{k}(\tau)$, and solving the partial differential equation for their generating function. We again do this for the case of a point sink singularity, to compare with the results of $\S 3.5$.

With the $\mathcal{M}_{k}$ defined as in $§ 3.5$, a similar analysis to that section (but using equations (4.11) and (4.12) instead of (3.8) and (3.9), and working with the scaled time $\tau$ ) gives

$$
\frac{d \mathcal{M}_{k}}{d \tau}= \begin{cases}\frac{Q f^{\prime}(0, \tau)}{2 A(\tau)} & k=0 \\ k\left(\mathcal{M}_{k-1}-\mathcal{M}_{k+1}\right) & k=1,2, \ldots\end{cases}
$$

Multiplying the equations for $k \geq 1$ by $\zeta^{k} / k$ and summing, we find

$$
\frac{\partial \mathcal{H}_{1}}{\partial \tau}+\left(1-\zeta^{2}\right) \frac{\partial \mathcal{H}_{1}}{\partial \zeta}=\mathcal{M}_{0} \zeta+\mathcal{M}_{1}
$$

where

$$
\mathcal{H}_{1}(\zeta, \tau)=\sum_{1}^{\infty} \frac{\mathcal{M}_{k}}{k} \zeta^{k} \equiv \mathcal{H}(1 / \zeta, \tau)
$$

Comparing (4.19) and (4.16), the solution is immediate from (4.17) as

$$
\begin{aligned}
\mathcal{H}_{1}(\zeta, \tau)=\int_{0}^{\tau} \mathcal{M}_{0}\left(\tau^{\prime}\right) \tanh \left(\tau^{\prime}-\tau+\right. & \left.\tanh ^{-1} \zeta\right) d \tau^{\prime} \\
& +\mathcal{H}_{10}\left(\frac{\zeta-\tanh \tau}{1-\zeta \tanh \tau}\right)+\int_{0}^{\tau} \mathcal{M}_{1}\left(\tau^{\prime}\right) d \tau^{\prime}
\end{aligned}
$$

where $\mathcal{H}_{10}(\zeta)=\mathcal{H}_{1}(\zeta, 0)$. In line with the comments following equation (4.15), the solution for the dipole problem would be exactly the same but with $\mathcal{M}_{0}\left(\tau^{\prime}\right)$ in the first integrand replaced by $\mathcal{M}_{0}+M \pi / A\left(\tau^{\prime}\right)$.

The function $\mathcal{H}_{1}(\zeta, \tau)$ is a generating function for the moments, which can now be 
recovered. Setting $\zeta=0$ in (4.20) gives a 'consistency condition' which must be satisfied, namely

$$
\mathcal{H}_{1}(0, t)=0=\int_{0}^{\tau} \mathcal{M}_{0}\left(\tau^{\prime}\right) \tanh \left(\tau^{\prime}-\tau\right) d \tau^{\prime}+\mathcal{H}_{10}(-\tanh \tau)+\int_{0}^{\tau} \mathcal{M}_{1}\left(\tau^{\prime}\right) d \tau^{\prime} .
$$

This equation, and the $k=0$ equation of (4.18), provide constraints on $\mathcal{M}_{0}(\tau), \mathcal{M}_{1}(\tau)$, and $A(\tau)$.

\subsection{A dipole in a circle}

In this section we illustrate by example how the results of $\S 4.3$ may be used to tackle initial-value problems. We consider ZST Stokes flow with a dipole placed at the centre of an initially-circular fluid domain ( $c f$. §3.6). A solution is first found with $\phi(0, t)=0$, but the lack of symmetry means that it is 'moving singularity' solution (or does not conserve momentum). We then consider the more difficult case $\phi(0, t) \neq 0$.

\subsubsection{The case $\phi(0, t)=0$}

We begin by finding the solution under the assumption that $\phi(0, t)=0$. The working here parallels that of $\S 3.6$ exactly, and we give only an outline. For a dipole of strength $M$ at the origin the Goursat function $\chi$ has the singular behaviour

$$
\chi(z, t)=\frac{M}{z}+O(1) \quad \text { as } z \rightarrow 0 ;
$$

and following through the steps leading to (3.26) we find that

$$
H_{e}^{\prime}(\zeta, t)=\frac{r^{2}}{\zeta}+\frac{2 M \theta_{3}(t)}{\zeta^{2}} \equiv\left[f^{\prime}(\zeta, t) \bar{f}(1 / \zeta, t)\right]_{e},
$$

where $\theta_{3}$ is defined by

$$
\theta_{3}(t)=\int_{0}^{t} \frac{d t^{\prime}}{f^{\prime}\left(0, t^{\prime}\right)}
$$

Thus in this case the conformal map must be a quadratic (rather than a cubic) polynomial,

$$
f(\zeta, t)=a(t) \zeta+b(t) \zeta^{2}
$$

where we fix $a(0)=1$ and $b(0)=0$. Matching the singularity at the origin in (3.8) (with $\gamma=0)$ gives the evolution equations

$$
\begin{array}{r}
\frac{d}{d t}(a b)=\frac{2 M}{a^{2}}, \\
\frac{d}{d t}\left(a^{2}+2 b^{2}\right)=0 .
\end{array}
$$

We solve these by setting $a(t)=\sin \theta(t)$; then $b(t) \sqrt{2}=\cos \theta(t)$ and the evolution is determined by the equation

$$
\theta-\sin 2 \theta+\frac{1}{4} \sin 4 \theta=-8 M t \sqrt{2}+\frac{\pi}{2} .
$$




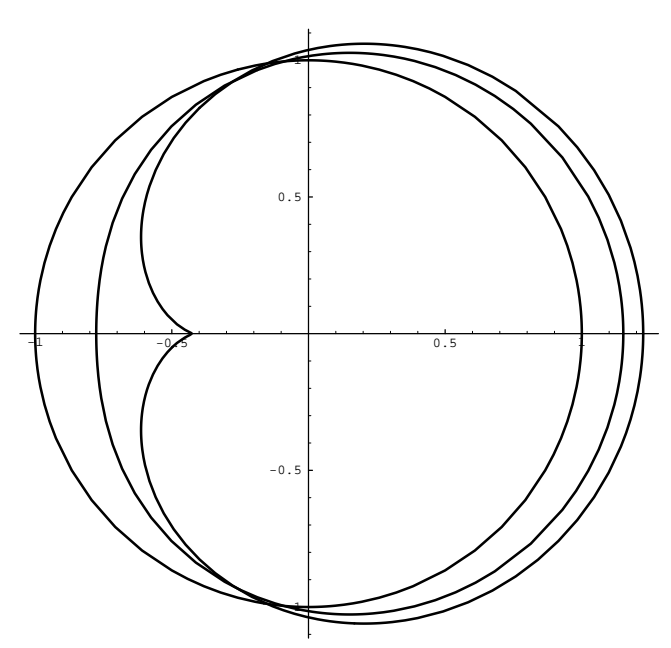

FIGURE 4. Evolution of a circular region containing a dipole at the origin.

As in $\S 3.6$ this solution also blows up via cusp formation, this time at $t=(\pi+4) /(32 M \sqrt{3})$. The evolution until the blow-up time is shown in Figure 4. Regarding the dipole as fixed, it is easy to see from the figure that the fluid domain has a nonzero net momentum along the positive $x$-axis. Its magnitude is

$$
\frac{4 \pi M b^{2}}{a\left(2 b^{2}-a^{2}\right)} \equiv \frac{2 \pi M \cos \theta}{\tan \theta \cos 2 \theta} .
$$

Alternatively, if the momentum is to be kept at zero, the dipole must move along the $x$-axis with the appropriate velocity.

\subsubsection{The case $\phi(0, t) \neq 0$.}

We now attempt to remedy the time-varying momentum (or imposed singularity motion) by allowing $\phi(0, t)$ to be nonzero, in line with the comments of $\S 3$, using the theory developed in $\S 4.3$.

The initial conformal map is just

$$
f(\zeta, 0)=a_{0} \zeta
$$

so by (3.13)

$$
H_{e}^{\prime}(\zeta, 0)=\left[f^{\prime}(\zeta, 0) \bar{f}(1 / \zeta, 0)\right]_{e}=\frac{a_{0}}{\zeta}
$$


and using (4.14) (noting that the area $\mathcal{M}_{0}=\pi a_{0}^{2}$ is constant for a dipole singularity),

$$
\mathcal{H}^{\prime}(\zeta, 0)=\frac{\mathcal{M}_{0}}{\zeta}-\pi H_{e}^{\prime}(\zeta, 0)=0
$$

so

$$
\mathcal{H}(\zeta, 0)=0
$$

As noted below equation (4.16), we now have $\hat{\mathcal{X}}_{e}(\zeta, \tau)=M /\left(\zeta f^{\prime}(0, \tau)\right)$, so if we define $\hat{\mathcal{M}}_{0}(\tau)$ by

$$
\hat{\mathcal{M}}_{0}(\tau)=\mathcal{M}_{0}+\frac{\pi M}{A(\tau)} \equiv \pi a_{0}^{2}+\frac{\pi M}{A(\tau)},
$$

the partial differential equation governing the evolution of $\mathcal{H}$ is exactly (4.16), with $\mathcal{M}_{0}$ replaced by $\hat{\mathcal{M}}_{0}$. With the above initial condition, the solution (from (4.17)) is

$$
\mathcal{H}(\zeta, \tau)=\int_{0}^{\tau} \frac{\hat{\mathcal{M}}_{0}\left(\tau^{\prime}\right) d \tau^{\prime}}{\tanh \left(\tau^{\prime}-\tau+\tanh ^{-1} \zeta\right)}+\int_{0}^{\tau} \mathcal{M}_{1}\left(\tau^{\prime}\right) d \tau^{\prime}
$$

Hence the solution for $H_{e}(\zeta, \tau)$ follows from (4.14) as

$$
H_{e}(\zeta, \tau)=a_{0}^{2} \log \zeta-\frac{1}{\pi} \int_{0}^{\tau} \frac{\hat{\mathcal{M}}_{0}\left(\tau^{\prime}\right) d \tau^{\prime}}{\tanh \left(\tau^{\prime}-\tau+\tanh ^{-1} \zeta\right)}-\frac{1}{\pi} \int_{0}^{\tau} \mathcal{M}_{1}\left(\tau^{\prime}\right) d \tau^{\prime}
$$

Differentiating with respect to $\zeta$, and making the substitution $x=\tanh \left(\tau-\tau^{\prime}\right)$ in the integral yields

$$
\begin{aligned}
H_{e}^{\prime}(\zeta, \tau) & =\frac{a_{0}^{2}}{\zeta}+a_{0}^{2} \int_{0}^{\tanh \tau} \frac{d x}{(\zeta-x)^{2}}+M \int_{0}^{\tanh \tau} \frac{d x}{A\left(\tau-\tanh ^{-1} x\right)(\zeta-x)^{2}} \\
= & -\frac{M}{A(\tau) \zeta}+\frac{\left(a_{0}^{2}+M / A(0)\right)}{\zeta-\tanh \tau}-\int_{0}^{\tanh \tau} \frac{M A^{\prime}\left(\tau-\tanh ^{-1} x\right) d x}{A^{2}\left(\tau-\tanh ^{-1} x\right)(\zeta-x)\left(1-x^{2}\right)}
\end{aligned}
$$

where we integrated by parts between the first and second equalities. As in the simpler example of $\S 3.6$, this must give us the singularities of $\bar{f}(1 / \zeta, \tau)$ within the unit disc. The above expression reveals these to be simple poles at $\zeta=0, \zeta=\tanh \tau$, joined by a line singularity along $(0, \tanh \tau)$ on the positive real $\zeta$-axis. Hence the form of the mapping function must be such that

$$
\bar{f}(1 / \zeta, \tau)=\frac{a(\tau)}{\zeta}+\frac{b(\tau)}{(\zeta-\tanh \tau)}+\int_{0}^{\tanh \tau} \frac{D(x, \tau)}{\zeta-x} d x
$$

so that

$$
f(\zeta, \tau)=a(\tau) \zeta+\frac{b(\tau) \zeta}{1-\zeta \tanh \tau}+\int_{0}^{\tanh \tau} \frac{\zeta D(x, \tau)}{1-x \zeta} d x
$$

for some unknown functions $a(\tau), b(\tau), D(x, \tau)$ (real, as the fluid domain is symmetric about the $x$-axis). We need to match singularities for this mapping function in the relation given in (3.26),

$$
H_{e}^{\prime}(\zeta, \tau)=\left[f^{\prime}(\zeta, \tau) \bar{f}(1 / \zeta, \tau)\right]_{e}
$$


The points $\zeta=0, \zeta=\tanh \tau$ give

$$
\begin{aligned}
f^{\prime}(0, \tau) & =-\frac{M}{A(\tau)}, \\
f^{\prime}(\tanh \tau, \tau) b(\tau) & =a_{0}^{2}+\frac{M}{A(0)} .
\end{aligned}
$$

We also need to match the line singularity at each point $\beta \in(0, \tanh \tau)$ (in a manner consistent with the above at the endpoints). Consideration of the leading-order behaviour on both sides of (4.26) using (4.24) and (4.25) gives

$$
f^{\prime}(\beta, \tau) D(\beta, \tau)=-\frac{M A^{\prime}\left(\tau-\tanh ^{-1}\left(\tau-\tanh ^{-1} \beta\right)\right)}{A^{2}\left(\tau-\tanh ^{-1} \beta\right)\left(1-\beta^{2}\right)} .
$$

We have to determine the real functions $a(\tau), b(\tau), D(x, \tau)$ and $A(\tau)$. We have three real relations $(4.27),(4.28)$ and (4.29); we also have the condition of zero net momentum,

$$
\iint_{\Omega(t)}\left(u_{1}+i u_{2}\right) d x d y=0
$$

which by symmetry is also real. In this expression, the complex velocity field $u_{1}+i u_{2}$ (given by (3.6)) is readily expressed in terms of the conformal mapping parameters. It is clear that the system of equations we have to deal with is formidable, and further analytical progress seems unlikely, although it might form the basis of a numerical approach.

\subsection{The Baiocchi transformation and moments with surface tension}

\subsubsection{The Baiocchi transformation}

In this section we revert to the simplified case $\phi(0, t)=0$ and briefly describe the rather complicated extension of the Baiocchi transform to the NZST Stokes problem. Guided by (3.8) and (3.9), we introduce a further complex variable $\tilde{\zeta}$ which, treated as a function of $\zeta$ and $t$, is given implicitly by the solution of the Löwner-Kufarev ordinary differential equation

$$
\frac{d \zeta}{d t}=-\frac{\gamma \zeta}{2 \mu} G_{+}(\zeta, t), \quad \zeta(0)=\tilde{\zeta}
$$

where for the purposes of this discussion $G_{+}(\zeta, t)$ is to be regarded as known. Performing a hodograph transformation, equation (4.30) corresponds to the partial differential equation

$$
\frac{\partial \tilde{\zeta}}{\partial t}-\frac{\gamma}{2 \mu} \zeta G_{+}(\zeta, t) \frac{\partial \tilde{\zeta}}{\partial \zeta}=0 . \quad \tilde{\zeta}(\zeta, 0)=\zeta
$$

for $\tilde{\zeta}(\zeta, t)$. Recalling that $\Re G_{+}(\zeta, t)>0$, the real part of this equation states that the the normal velocity in the $\tilde{\zeta}$-plane of the image of $|\zeta|=1$ is positive; that is, the domain corresponding to $\Omega(t)$ in that plane is expanding.

Let us write

$$
f(\zeta, t)=\tilde{f}(\tilde{\zeta}, t),
$$

and similarly for other variables regarded as functions of $\tilde{\zeta}$ and $t$ instead of $\zeta$ and $t$. It 
follows from (3.9) that

$$
\frac{\partial \tilde{f}}{\partial t}(\tilde{\zeta}, t)=2 \widetilde{\Phi}(\tilde{\zeta}, t)
$$

and from (3.8) that

$$
\frac{\partial \widetilde{H}}{\partial t}(\tilde{\zeta}, t)=-2 \widetilde{\mathcal{X}}(\tilde{\zeta}, t)
$$

choosing the arbitrary function of time appropriately. Equation (4.32) is the NZST generalisation of (3.14).

The analysis of $\S 3.4$ now goes through almost unaltered, yielding

$$
\frac{\partial u}{\partial t}=-\tilde{\mathcal{A}}(\tilde{\zeta}, t)
$$

the derivative being at fixed $\tilde{\zeta}$; this is the generalisation of (3.15). Singularities are thus fixed in the $\tilde{\zeta}$-plane.

\subsubsection{The Schwarz function}

Let us for the sake of completeness outline some properties of the Schwarz function for NZST problems. It is easy to deduce from (4.10) that

$$
\frac{\partial g}{\partial t}(z, t)+2 \frac{\partial}{\partial z}(\phi(z, t) g(z, t))=-2 \frac{\partial \chi}{\partial z}(z, t)
$$

in which $\gamma$ does not appear explicitly. If $\chi(z, t)$ and $\phi(z, t)$ are regarded as known, the variable $\tilde{\zeta}$ introduced above is constant along the characteristics of (4.34), namely

$$
\frac{d z}{d t}=2 \phi(z, t)
$$

as may be seen from (3.9) and (4.30). Since singularities are fixed in the $\tilde{\zeta}$-plane, equation (4.35) governs how they move in the $z$-plane. In the ZST case, in which $\tilde{\zeta} \equiv \zeta$, equation (4.35) implies that on the moving boundary $\bar{z}=g(z, t)$, the fluid particles move with the characteristic velocity (see equation (4.6)).

Equation (4.34) implies that the primitive $h(z, t)$ of the Schwarz function satisfies

$$
\frac{\partial h}{\partial t}(z, t)+2 \phi(z, t) \frac{\partial h}{\partial z}(z, t)=-2 \chi(z, t),
$$

which is equivalent to (4.32). The corresponding results for ZST Hele-Shaw flow are (cf. equation (2.9))

$$
\frac{\partial g}{\partial t}(z, t)=2 \frac{\partial w}{\partial z}(z, t), \quad \frac{\partial h}{\partial t}(z, t)=2 w(z, t) .
$$

In the Stokes flow case, we may define an artificial velocity field, which coincides with the true velocity on the moving boundary, by introducing an artificial analytic velocity potential $W^{*}(z, t)$ via the equation

$$
\frac{\partial W^{*}}{\partial z}(z, t)=2 \bar{\phi}(g(z, t), t)+i \frac{\gamma}{2 \mu}\left(\frac{\partial g}{\partial z}(z, t)\right)^{\frac{1}{2}}
$$


(see equation (4.6)). It then follows that

$$
\frac{\partial g}{\partial t}=\frac{\partial}{\partial z}\left(W^{*}(z, t)-\bar{W}^{*}(g(z, t), t)\right), \quad \frac{\partial h}{\partial t}=\left(W^{*}(z, t)-\bar{W}^{*}(g(z, t), t)\right) .
$$

In this way, we can define an 'equivalent' ZST Hele-Shaw flow for a given ZST or NZST Stokes flow, as the Hele-Shaw flow with the same moving boundary. Its velocity potential is just

$$
w(z, t)=\frac{1}{2}\left(W^{*}(z, t)-\bar{W}^{*}(g(z, t), t)\right) ;
$$

note that this is pure imaginary on $\bar{z}=g(z, t)$, as required.

\subsubsection{The moments}

The relevant conserved quantities for the NZST problem are easily derived from the Baiocchi-transformed problem. The moments are

$$
\widetilde{\mathcal{M}}_{k}(t)=\iint_{\Omega(t)} \tilde{\zeta}^{k} d x d y
$$

though the integrand in (4.37) can be generalised to any analytic function of $\tilde{\zeta}$; generalisations of this kind may again be useful for rational maps (cf. [8]). We may, as before, derive the result

$$
\frac{d \widetilde{\mathcal{M}}_{k}(t)}{d t}=Q \delta_{0 k}
$$

for flows driven by a single point source/sink at the origin. (Note from (4.30) that $\zeta=0$ corresponds to $\tilde{\zeta}=0$ for all $t$.) If we write

$$
\tilde{\zeta}^{k}=\zeta^{k} \sum_{l=0}^{\infty} a_{k, l}(t) \zeta^{l}, \quad a_{k, l}(0)=\delta_{0 l}
$$

then, as

$$
\frac{\partial \tilde{\zeta}}{\partial t}-\frac{\gamma}{2 \mu} \zeta G_{+}(\zeta, t) \frac{\partial \tilde{\zeta}}{\partial \zeta}=0
$$

we have

$$
\frac{d a_{k, l}}{d t}=\frac{\gamma}{2 \mu} \sum_{m=0}^{l} \frac{G_{+}^{(m)}(0, t)}{m !}(k+l-m) a_{k, l-m}
$$

Since

$$
\begin{aligned}
\frac{d}{d t} \iint_{\Omega(t)} \tilde{\zeta}^{k} d x d y & =\sum_{l=0}^{\infty}\left(\frac{d a_{k, l}}{d t} \mathcal{M}_{k+l}+a_{k, l} \frac{d \mathcal{M}_{k+l}}{d t}\right) \\
& =\sum_{m=k}^{\infty} a_{k, m-k}\left(\frac{d \mathcal{M}_{m}}{d t}+\frac{m \gamma}{2 \mu} \sum_{r=0}^{\infty} \frac{G_{+}^{(r)}(0, t)}{r !} \mathcal{M}_{m+r}\right)
\end{aligned}
$$

we recover (3.22). However (4.38) rather than (3.22) is evidently the most concise moment formulation in the NZST case. 
For the polynomial example with $f(\zeta, t)$ given by $(2.5)$ the $\tilde{\zeta}$-moments are easy to calculate. For since $\zeta$ and $\tilde{\zeta}$ coincide at time $t=0$ we have

$$
\widetilde{M}_{k}(0)=\iint_{\Omega(0)} \tilde{\zeta}^{k}(z, 0) d x d y \equiv \iint_{\Omega(0)} \zeta^{k}(z, 0) d x d y=\mathcal{M}_{k}(0),
$$

that is, the $\tilde{\zeta}$-moments and the usual moments coincide at $t=0$. The usual moments were found in $\S 3.5$, hence

$$
\widetilde{\mathcal{M}}_{0}(0)=\pi a^{2}(0)\left(1+\frac{b^{2}(0)}{n}\right), \quad \widetilde{\mathcal{M}}_{n-1}(0)=-\frac{\pi a^{2}(0) b(0)}{n},
$$

and all other $\widetilde{\mathcal{M}}_{k}(0)$ vanish. Thus, by $(4.38)$,

$$
\begin{gathered}
\widetilde{\mathcal{M}}_{0}(t)=\pi a^{2}(0)\left(1+\frac{b^{2}(0)}{n}\right)+Q t, \quad \widetilde{\mathcal{M}}_{n-1}(t)=-\frac{\pi a^{2}(0) b(0)}{n}, \\
\widetilde{\mathcal{M}}_{k}(t) \equiv 0 \quad k \neq 0, n-1 .
\end{gathered}
$$

However, from (4.30),

$$
\tilde{\zeta} \sim \zeta \exp \left(\frac{\gamma}{2 \mu} \int_{0}^{t} G_{+}(0, \tau) d \tau\right) \quad \text { as } \quad \zeta \rightarrow 0 ;
$$

and since

we have

$$
\widetilde{\mathcal{M}}(t)=\frac{1}{2 i} \oint_{|\zeta|=1} \tilde{\zeta}^{k} f^{\prime}(\zeta, t) \bar{f}(1 / \zeta, t) d \zeta
$$

$\widetilde{\mathcal{M}}_{0}(t)=\pi a^{2}(t)\left(1+\frac{b^{2}(t)}{n}\right), \quad \widetilde{\mathcal{M}}_{n-1}(t)=-\frac{\pi a^{2}(t) b(t)}{n} \exp \left(\frac{(n-1) \gamma}{2 \mu} \int_{0}^{t} G_{+}(0, \tau) d \tau\right)$,

which, with (4.40) completely specify the motion.

It is also instructive to consider, for ZST Hele-Shaw flow and both ZST and NZST Stokes flow, the evolution of solutions given by rational maps of the form

$$
f(\zeta, t)=\sum_{n=1}^{N} \frac{\bar{A}_{n}(t)}{1-\bar{a}_{n}(t) \zeta}
$$

with $\left|a_{n}\right|<1$. Maps of this form have been used to describe viscous sintering [20, 21, 44]. The map (4.41) contains $2 N$ unknowns; $N$ equations for these can be written down straight away using our results for singularity dynamics. Since

$$
\bar{f}(1 / \zeta, t)=\sum_{n=1}^{N} \frac{A_{n}(t) \zeta}{\zeta-a_{n}(t)},
$$

the singularities of $u$ in $|\zeta|<1$ are located at $\zeta=a_{m}(t), m=1,2, \ldots, N$. We thus have:

$$
\begin{array}{rlrl} 
& \text { ZST Hele-Shaw } & z_{m}(t) & =\sum_{n=1}^{N} \frac{\bar{A}_{n}(t)}{1-\bar{a}_{n}(t) a_{m}(t)} \text { is constant; } \\
& \text { ZST Stokes } & a_{m}(t) \text { is constant; } \\
\text { NZST Stokes } & \frac{d a_{m}}{d t} & =-\frac{\gamma a_{m}}{2 \mu} G_{+}\left(a_{m}, t\right)
\end{array}
$$


(here the $t$-dependence in $G_{+}(\zeta, t)$ is shorthand for its dependence on all of the $a_{n}(t)$, so (4.44) is a coupled system).

The second set of $N$ equations in each case can also be written down at once, this time using the moments

$$
\frac{1}{2 i} \oint_{|\zeta|=1} \xi^{k} f^{\prime}(\zeta, t) \bar{f}(1 / \zeta, t) d \zeta, \quad k=0,1,2, \ldots
$$

where

$$
\xi(\zeta, t)= \begin{cases}z=f(\zeta, t) & (\text { Hele-Shaw) } \\ \zeta & (\text { ZST Stokes) } \\ \tilde{\zeta}(\zeta, t) & \text { (NZST Stokes) }\end{cases}
$$

so that, by the constancy of singularities just mentioned, $\xi\left(a_{m}(t), t\right)=\xi_{m}$, say, is independent of $t$. If no driving singularities are present (in the absence of surface tension no motion will then occur), all the moments are constant and by a residue calculation we see that

$$
\sum_{m=1}^{N} \xi_{m}^{k} A_{m} a_{m} \sum_{n=1}^{N} \frac{\bar{a}_{n} \bar{A}_{n}}{\left(1-\bar{a}_{n} a_{m}\right)^{2}} \quad \text { is constant, }
$$

so that, recalling that the $\xi_{m}$ are constant,

$$
A_{m} a_{m} \sum_{n=1}^{N} \frac{\bar{a}_{n} \bar{A}_{n}}{\left(1-\bar{a}_{n} a_{m}\right)^{2}} \quad \text { is also constant, } m=1, \ldots, N .
$$

(With a driving singularity, the $\xi_{m}$ remain constant but there is time dependence in (4.45).) Summing (4.46) over $m$ shows that it in fact represents only $2 N-1$ real equations. For Hele-Shaw the $a_{n}$ are determined only up to a common factor $e^{i \nu(t)}$, where $\nu$ is real and corresponds to the invariance under $\zeta$ rotations, while for Stokes the $A_{n}$ instead exhibit this indeterminacy, corresponding to invariance under $z$-rotations.

This example illustrates some similarities and differences between the models: (4.46) is the same in all three cases but (4.44) is significantly more complicated than (4.42) or (4.43). It also illustrates the complementary roles of the conserved quantities and the singularity dynamics.

We note from (4.30) that, taking $\tilde{\zeta}$ fixed,

$$
\frac{d}{d t} \ln |\zeta|=-\frac{\gamma}{2 \mu} \frac{1}{\left|f^{\prime}(\zeta, t)\right|}<0 \quad \text { on }|\zeta|=1,
$$

provided that $\gamma>0$. This means that the boundary of the fluid domain $|\zeta|<1$ always moves outwards in the $\tilde{\zeta}$-plane, starting from the unit circle at $t=0$. This is in accordance with the interpretation of (4.30) as a Löwner-Kufarev equation; a similar interpretation for the ZST Hele-Shaw problem is given in [19].

Lastly we note that we have chosen here, for brevity of exposition, to define the variable $\tilde{\zeta}$ via (4.30). It may instead be derived by seeking a variable in which singularities are fixed and moments conserved. In the ZST case, such a calculation identifies $\zeta$, the variable originally introduced for the different purpose of mapping the free boundary to a fixed one. The fact that $\zeta$ simultaneously performs these two distinct rôles indicates some of the simplifying structure inherent in the ZST Stokes flow problem. 


\section{6 'Weak' solutions in Stokes flow}

We saw from the example of $\S 3.2$ (the point sink driven case) that whereas solutions to the ZST problem frequently undergo finite-time blow-up via cusp formation in the free boundary (at time $t^{*}$, say), inclusion of nonzero surface tension $\gamma$, however small, can prevent this. This gives rise to the question of what happens as $\gamma$ becomes vanishingly small. Clearly, setting $\gamma=0$ in (3.8) and (3.9) is the appropriate limit for times $t<t^{*}$ when the free boundary is smooth. However, as $t \rightarrow t^{*}$ and a zero (or zeros) of $f^{\prime}(\zeta, t)$ approaches the unit circle (at $\zeta=\zeta^{*}$, say) the function $G_{+}(\zeta, t)$ tends to infinity as $(\zeta, t) \rightarrow\left(\zeta^{*}, t^{*}\right)$. We thus have "zero times infinity" in (3.8) and (3.9), hence a singular limit in these equations.

We shall assume that the moving boundary of the NZST problem (and so of its limit as $\gamma \rightarrow 0^{+}$) does not intersect itself for any $\gamma$. To see what the appropriate modification to the system is, we return to the kinematic boundary condition in the form (4.7) which says

$$
\Re\left(\frac{1}{\zeta f^{\prime}(\zeta, t)}\left[2 \Phi(\zeta, t)-\frac{\partial f}{\partial t}(\zeta, t)\right]\right)=-\frac{\gamma}{2 \mu} \frac{1}{\left|f^{\prime}(\zeta, t)\right|} \quad \text { on }|\zeta|=1 .
$$

In the NZST case and with $\Phi(0, t)=0$ (which we assume throughout this section), the function on the left-hand side of (4.48) is analytic in the unit disc; Poisson's formula then applies, leading to (3.9). As $t \rightarrow t^{*}$ however, we note that the function on the right-hand side of (4.48) tends to zero as $\gamma \rightarrow 0$, except at the points $\zeta^{*}$ such that $f^{\prime}\left(\zeta^{*}, t^{*}\right)=0$, where we have a zero times infinity. This situation represents a persistent cusp in the free boundary. We deduce that in the limit $\gamma \rightarrow 0$, equation (3.9) should be replaced by

$$
\frac{1}{\zeta f^{\prime}(\zeta, t)}\left(2 \Phi(\zeta, t)-\frac{\partial f}{\partial t}(\zeta, t)\right)=-\sum_{j} G_{j}(t)\left(\frac{\zeta_{j}^{*}+\zeta}{\zeta_{j}^{*}-\zeta}\right),
$$

where $j$ labels the points $\zeta_{j}^{*}$ on the unit disc that correspond to the points at which $\partial \Omega(t)$ is not smooth, assumed to be finite in number. (A similar idea is introduced for Hele-Shaw flows in [19].) The functions $G_{j}(t)$ are to be determined but are real and satisfy

$$
G_{j}(t) \equiv 0 \quad \text { for } t<t_{j}^{*} .
$$

The functions $G_{j}(t)$ act as 'switches' for the singularities on the right-hand side of (4.49): for $0 \leq t<t^{*}=\min _{j}\left\{t_{j}^{*}\right\}$, the free boundary is smooth, the first cusps appear at time $t^{*}$, and thereafter more cusps may appear (and persist) as the $G_{j}(t)$ are switched on. The non-smooth nature of $\partial \Omega(t)$ when any of the $G_{j}(t)$ are active motivates our terminology 'weak solutions'. Equation (4.49) is clearly consistent with (4.48).

An alternative way to derive (4.49) is to consider the definition of $G_{+}(\zeta, t)$, equation (3.10), and argue that in the limit $\gamma \rightarrow 0^{+}$we have

$$
\frac{\gamma}{2 \mu} G_{+}(\zeta, t) \mapsto \sum_{j} G_{j}(t)\left(\frac{\zeta_{j}^{*}+\zeta}{\zeta_{j}^{*}-\zeta}\right),
$$

where again the $G_{j}(t)$ switch on at cusp formation times $t_{j}^{*}$. Whichever way we argue 
the result is the same, and equation (3.8) is replaced by

$$
2 \mathcal{X}^{\prime}(\zeta, t)+\frac{\partial}{\partial t}\left(f^{\prime}(\zeta, t) \bar{f}(1 / \zeta, t)\right)=\sum_{j} G_{j}(t) \frac{\partial}{\partial \zeta}\left(\left(\frac{\zeta_{j}^{*}+\zeta}{\zeta_{j}^{*}-\zeta}\right) \zeta f^{\prime}(\zeta, t) \bar{f}(1 / \zeta, t)\right)
$$

To illustrate, consider the example of $\S 3.2$ in this limit. Recall that the mapping is

$$
z=f(\zeta, t)=a(t)\left(\zeta-\frac{b(t)}{n} \zeta^{n}\right)
$$

where in the NZST evolution

$$
\frac{d}{d t}\left(a^{2}\left(1+\frac{b^{2}}{n}\right)\right)=\frac{Q}{\pi}, \quad \frac{d}{d t}\left(a^{2} b\right)=-\frac{\gamma}{\pi \mu}(n-1) a b K(b) .
$$

For $t<t^{*}=(-\pi / Q) a_{0}^{2}\left(1-b_{0}\right)\left(1-b_{0} / n\right)$ the evolution for $\gamma=0^{+}$is as for ZST theory; at time $t^{*}, b$ reaches the value 1 and $(n-1)$ cusps form in the free boundary at the points

$$
\zeta_{j}^{*}=\exp \left(\frac{2 \pi i j}{n-1}\right) \quad 1 \leq j \leq n-1 .
$$

The functions $G_{j}(t)$ then 'switch on' and we must match singularities at the points $\zeta=\zeta_{j}^{*}$ and $\zeta=0$ in (4.51). There is no singularity at $\zeta=\zeta_{j}^{*}$ on the left-hand side, so we must eliminate that on the right-hand side, giving

$$
f^{\prime}\left(\zeta_{j}^{*}, t\right)=0 \quad t \geq t^{*}
$$

from which

$$
b(t) \equiv 1 \quad t \geq t^{*},
$$

so persistent cusps occur in the free boundary. Near $\zeta=0$, matching singularities at orders $\zeta^{-n}$ and $\zeta^{-1}$ as in $\S 3.2$, and assuming on the grounds of symmetry that the functions $G_{j}(t)$ are all equal in this case, gives for $t>t^{*}$

$$
\frac{d}{d t}\left(a^{2}\right)=\frac{n Q}{\pi(n+1)}, \quad \frac{d}{d t}\left(a^{2}\right)=-a^{2}(n-1)^{2} G(t) .
$$

The first of these equations corresponds to the first of (4.52). The latter can be verified against the $\gamma \rightarrow 0$ limit of the second of (4.52), giving the function $G(t)$ as

$$
G(t)=-\frac{Q}{(n-1)^{2} S(t)}
$$

where $S(t)$ is the area of the fluid domain.

We may also investigate the fluid velocity in the neighbourhood of the cusps predicted by this model using (3.6), since we have explicit expressions for $\Phi(\zeta, t)$ and $\mathcal{X}(\zeta, t)$. For the point $\zeta=\zeta_{j}^{*}+\epsilon$ within the fluid (where $\varepsilon$ is complex and $|\epsilon| \ll 1$ ) the result is

$$
u_{1}+i u_{2}=\frac{\partial f}{\partial t}\left(\zeta_{j}^{*}, t\right)-2 G(t) a(n-1) \zeta_{j}^{*}+O(\epsilon)
$$

the first term here is the velocity of the cusp itself as a point of the free boundary, and the second term represents slip of the fluid past the cusp, $G(t)$ providing a measure of the degree of slip (see [27] for a discussion of this point for a steady flow).

In general terms, then, each time a cusp forms a new unknown function of time appears, 
representing the corresponding 'zero times infinity'. At the same time, we have the new condition that $f^{\prime}\left(\zeta_{j}^{*}, t\right)=0$ for the relevant $\zeta_{j}^{*}$, and so we have one new complex equation, in addition to the singularity-matching equations at the origin, from which to determine the two real quantities $G_{j}(t)$ and $\arg \zeta_{j}^{*}(t)$. In contrast with the 'slit' model of [19] for Hele-Shaw flows, the Stokes flow weak solutions are thus fully determined.

It can easily be shown $[\mathbf{2 5}]$ that the approach just described is consistent with the limit as $\gamma \rightarrow 0^{+}$of the explicit solution with $\gamma>0$. To investigate this limit further, we now use the Baiocchi transform (in terms of $\tilde{\zeta}$ ), derived above for $\gamma>0$. When $\gamma=0$, it follows from (4.47) that, away from a cusp,

$$
\frac{d}{d t}|\zeta|=0 \quad \text { on }|\zeta|=1
$$

with $\zeta(0)=\tilde{\zeta}$; thus, points with $|\zeta|=1$ remain on $|\tilde{\zeta}|=1$ for all $t>0$ (when $\gamma>0$ they move outwards in the $\tilde{\zeta}$-plane). However, as we shall see, after a cusp has formed these points do not fill the whole of $|\tilde{\zeta}|=1$. For brevity, let us consider a single cusp located at the image of $\zeta=1$, so that (4.50) becomes

$$
\frac{\gamma}{2 \mu} G_{+}(\zeta, t) \mapsto G(t) \frac{1+\zeta}{1-\zeta} .
$$

Writing $\zeta=e^{i \theta}, \tilde{\zeta}=e^{i \tilde{\theta}}$, we then have, from (4.47) that, on $|\zeta|=1$,

$$
\frac{d \theta}{d t}=-2 G(t) \cot \frac{\theta}{2}, \quad \theta=\tilde{\theta} \quad \text { at } \quad t=0,
$$

so that

$$
\cos \frac{\theta}{2}=\left(\cos \frac{\tilde{\theta}}{2}\right) \exp \int_{0}^{t} G(\tau) d \tau
$$

This shows that the preimage $|\zeta|=1$ of the moving boundary, namely $0 \leq \theta<2 \pi$, coincides with the sector $\pi-\widetilde{\Theta}(t) \leq \tilde{\theta}<\pi+\widetilde{\Theta}(t)$ of $|\tilde{\zeta}|=1$, where

$$
\widetilde{\Theta}(t)=2 \sin ^{-1} \exp \int_{0}^{t} G(\tau) d \tau
$$

where $0<\widetilde{\Theta}(t) \leq \pi$ and $\widetilde{\Theta}(t)$ is monotonic decreasing in $t$.

As already noted, points that are fixed in the $\tilde{\zeta}$-plane evolve in the $z$-plane according to (4.35); moreover, it follows from (3.6)-(3.7) that, for $\gamma=0$,

$$
u_{1}+i u_{2}=2 \phi(z, t) \quad \text { on } \partial \Omega(t)
$$

so fixed values of $\tilde{\theta}$ satisfying $\pi-\widetilde{\Theta}(t)<\tilde{\theta}<\pi+\widetilde{\Theta}(t)$ correspond to fixed fluid particles. Before cusp formation we have $\theta=\tilde{\theta}$, and fluid particles that start on the fluid surface remain there. After cusp formation, however, surface particles are able to enter the bulk of the fluid via the cusp, and this is the fate that has befallen those which were initially located at $\theta=\tilde{\theta}$ with $0 \leq \tilde{\theta}<\pi-\widetilde{\Theta}(t)$ or $\pi+\widetilde{\Theta}(t)<\tilde{\theta} \leq 2 \pi$. In the $\zeta$-plane, particles which started on the surface thus occupy both $|\zeta|=1$ and a slit which starts from $\zeta=1$ and lies in $|\zeta|<1$; if one follows the usual prescription that particles which start on the surface remain there, then the moving boundary in the physical domain contains a slit which emanates from the cusp. The slit forms because the velocity of the cusp is not equal to the local fluid velocity; points on the slit do move with the flow. 
A local analysis about the cusp shows that no points starting in $|\tilde{\zeta}|>1$ can enter the unit disc $|\zeta| \leq 1$, so the fluid domain is given for all $t$ by $|\tilde{\zeta}| \leq 1$. A formulation akin to (3.16) and (3.17) is then possible in the $\tilde{\zeta}$-plane, but it is a codimension-two moving boundary problem $[\mathbf{2 6}]$ involving the unknown $\widetilde{\Theta}(t)$, and with different boundary conditions holding on the two parts of $|\tilde{\zeta}|=1$ separated by the points $\tilde{\theta}=\pi \pm \widetilde{\Theta}(t)$.

We now make some of these statements more concrete. The crucial step in the weak solution is the substitution (4.50) in the governing equations. Making this substitution in equation (4.39) (which is equivalent to (4.30)), and assuming only one cusp at the image of $\zeta=1$ for simplicity, we find the equation satisfied by the variable $\tilde{\zeta}$ in this limit to be

$$
\frac{\partial \tilde{\zeta}}{\partial t}-\zeta \frac{\partial \tilde{\zeta}}{\partial \zeta} G(t)\left(\frac{1+\zeta}{1-\zeta}\right)=0
$$

It is convenient to define the scaled time $\tau$ by

$$
\frac{d t}{d \tau}=\frac{1}{G(t)},
$$

and to take the origin of time (both $t$ and $\tau$ ) to be the time of cusp formation; considering $\tilde{\zeta}$ as a function of $\zeta$ and $t$ (or $\tau$ ) we then require $\tilde{\zeta}(\zeta, 0)=\zeta$ since the two variables are identical for times prior to cusp formation in the ZST problem. We can now write down the solution of (4.56) as

$$
\tilde{\zeta}=\mathcal{K}^{-1}\left(\mathcal{K}(\zeta) e^{\tau}\right),
$$

where $\mathcal{K}(\cdot)$ denotes the Koebe map of univalent function theory [12]. It is defined by $\mathcal{K}(\zeta)=\zeta /(1+\zeta)^{2}$, and maps $|\zeta|<1$ onto the whole complex plane, minus the semi-infinite line segment $(-\infty,-1 / 4]$.

For this simple case (4.57) gives the exact relation between $\zeta$ and $\tilde{\zeta}$ in terms of $\tau$. The domain in $\tilde{\zeta}$-space corresponding to the unit disc in $\zeta$-space is illustrated in Fig. 5 for a particular time $\tau$; it is again the unit disc but, as noted above, only part of the bounding unit circle now corresponds to the free boundary $\partial \Omega$, as is illustrated by the outer contour not extending fully round the circle. A concrete example is provided by the "limaçon" problem (the $n=2$ case of the solutions of $\S 3.2$, also considered in $\S 4.6$ above). $G(t)$ is given by (4.53) which, taking $S(0)=Q$ for simplicity, gives the relation between $t$ and $\tau$ as $1-t=e^{-\tau}$. For times $t \geq t^{*}$ the weak solution theory of $\S 4.6$ gives $b(t) \equiv 1$ (the cusp persists); and in terms of the $\tilde{\zeta}$-theory we have $\tilde{f}(\tilde{\zeta}, t)=f(\zeta, t)$. Thus

$$
\tilde{f}(\tilde{\zeta}, t)=a(t)\left(\zeta-\frac{\zeta^{2}}{2}\right), \quad \zeta=\mathcal{K}^{-1}((1-t) \mathcal{K}(\tilde{\zeta})),
$$

and the free boundary of the fluid domain is given by the image under $\tilde{f}$ of the relevant $\operatorname{arc}|\tilde{\zeta}|=1$ in $\tilde{\zeta}$-space, as in figure 5 . The ends $\tilde{\zeta}_{1}, \tilde{\zeta}_{2}$ of this arc are given by the images under (4.57) of the point $\zeta=1$ in $\tilde{\zeta}$-space, that is,

$$
\tilde{\zeta}_{j}=\mathcal{K}^{-1}\left(\frac{1}{4(1-t)}\right)=1-2 t \pm 2 i \sqrt{t(1-t)}
$$

extinction, with zero fluid area, occurs at $t=1$. The moments may also be recovered explicitly, using the definition (4.37).

Finally note that the time-irreversibility of NZST Stokes flows persists in the limit $\gamma \rightarrow$ 


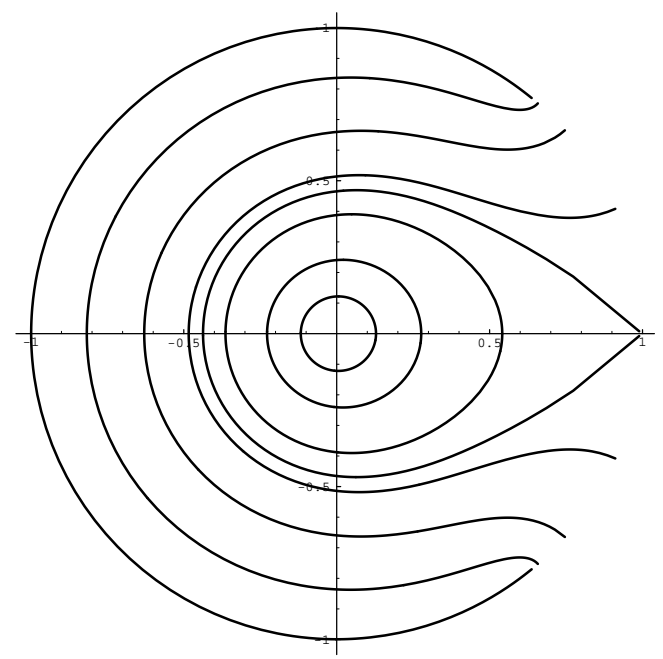

Figure 5. The domain in $\tilde{\zeta}$-space corresponding to the unit disc in $\zeta$-space, and to the fluid domain $\Omega$ in $z$-space, shown at time $\tau=0.2$. The contours illustrate the $\zeta \leftrightarrow \tilde{\zeta}$ correspondence; each corresponds to a different $r$-value for $|\zeta|=r$. Working inwards, the values shown are: $r=1,0.8,0.6,0.45,0.40275,0.33,0.2,0.1$.

$0^{+}$(before cusp formation, the ZST problem is time-reversible). Consider a sink-driven flow with a persistent cusp travelling towards the fluid. If the sink is instantaneously replaced by a source, the cusp immediately smooths out (as can be shown, for example, by analysing the limit $\gamma \rightarrow 0^{+}$), and in general does not reappear. (We must qualify this remark by noting that the lack of a maximum principle for Stokes flows means that we cannot guarantee that a cusp that is initially travelling inwards continues to do so. Indeed, it is possible in principle that cusps could appear then disappear at a later time.)

\section{Discussion}

\subsection{Gravity-driven Stokes flow}

Another way we might seek to extend the theory is by incorporating nonzero gravitational effects into flows. This has been considered by several authors for steady flows (see for instance $[\mathbf{1 7}, \mathbf{2 7}]$ ), but presents much more of a challenge in the time-dependent case, for reasons we now give.

The slow-flow equations are now

$$
\nabla p=\mu \nabla^{2} \mathbf{u}+\rho \mathbf{g}, \quad \nabla \cdot \mathbf{u}=0
$$

we again assume two-dimensional flow, with gravity acting in the $(x, y)$-plane along the negative $y$-axis, so that $\mathbf{g}=-g \mathbf{j}$. A reduced pressure $\hat{p}=p+\rho g y$ may be defined, with 
respect to which we get the usual Stokes flow equations, but with modified stress and kinematic boundary conditions. We again introduce the Goursat functions $\phi$ and $\chi$, so that the streamfunction $\psi$ is given by the imaginary part of (3.4). Omitting the details, the modified stress boundary condition (replacing (3.7)) turns out to be

$$
\phi(z, t)+z \overline{\phi^{\prime}(z, t)}+\overline{\chi^{\prime}(z, t)}=\frac{i \gamma}{2 \mu} \frac{d z}{d s}+\frac{i \rho g}{8 \mu}\left(-2 h(z, t)+z^{2}\right) \quad \text { on } \partial \Omega(t) .
$$

Here, as in $\S 3.3, h(z, t)$ is a primitive of the Schwarz function of the free boundary, so it is analytic in some neighbourhood of $\partial \Omega(t)$. Introducing the usual conformal map $f(\zeta, t)$, this can be recast in the $\zeta$-plane, and analytically continued away from the unit circle. Assuming zero surface tension for simplicity, the resulting equation is

$$
\Phi(\zeta, t)+f(\zeta, t) \frac{\bar{\Phi}^{\prime}(1 / \zeta, t)}{\bar{f}^{\prime}(1 / \zeta, t)}+\frac{\mathcal{X}^{\prime}(1 / \zeta, t)}{\bar{f}^{\prime}(1 / \zeta, t)}=\frac{i \rho g}{8 \mu}\left(-2 H(\zeta, t)+f^{2}(\zeta, t)\right),
$$

holding on $|\zeta|=1$, and elsewhere by analytic continuation (cf. equation (2.18) of [41]). The kinematic boundary condition may also be recast in the $\zeta$-plane, using techniques similar to those of $\S 4.2$ (and [41]). This leads to the condition

$$
\Re\left(\frac{1}{\zeta f^{\prime}(\zeta, t)}\left(2 \Phi(\zeta, t)-f_{t}(\zeta, t)-\frac{\rho g}{8 \mu}\left(2 H(\zeta, t)+f^{2}(\zeta, t)\right)\right)\right)=0 \quad \text { on }|\zeta|=1 .
$$

The function $H(\zeta, t)$ satisfies (3.13), and it has singularities within the unit disc corresponding to those of $\bar{f}(1 / \zeta)$. The boundary condition (5.1) is thus non-trivial to analytically continue away from the unit circle - the continuation will depend on which mapping function we choose.

\subsection{General remarks}

We have attempted to describe the theory of the Hele-Shaw and Stokes flow problems with the aim of emphasising the similarities and differences between them; they are set out at the end of Section 3. (For another view of this question, see [7] in this volume.) There is no doubt that Stokes flow leads to the more complicated structure in the zero-surfacetension case, but set against that is the fact that it is possible to construct solutions with non-zero surface tension; would that this were the case for the Hele-Shaw problem! We have also outlined some ways in which the theory for Stokes flow can be extended; we have not mentioned the many complications that arise when the fluid domain is multiply connected, but [6] describes some general results for multiply connected flows in $n$ dimensions, while [8] and [46] give explicit solutions using complex variable methods. Among the many other fascinating possibilities for future investigation, we single out in particular the idea of a weak solution, in which the free boundary to a zero-surfacetension problem has a persistent singularity. A proposal for the corresponding Hele-Shaw situation was made in [19], but here we have been able to put the weak solution for Stokes flow on a footing that is robust enough to justify more detailed investigation. 


\section{Acknowledgements}

We are grateful for helpful conversations with Dr J.R. Ockendon. The first author acknowledges financial support from the E.P.S.R.C. via a research studentship, and the third author is grateful for support from the Leverhulme Trust.

\section{References}

[1] Antanovskir. L.K. A plane inviscid bubble placed within a creeping viscous flow: formation of a cusped bubble. Eur. J. Mech. B 13, 491-509 (1994).

[2] Antanovskit. L.K. Quasi-steady deformation of a two-dimensional bubble placed within a potential viscous flow. Meccanica 29, 27-42 (1994).

[3] BAIOCCHI, C. Variational and quasivariational inequalities: applications to free boundary problems. Wiley (1984).

[4] Buckmaster, J.D. Pointed bubbles in slow viscous flow. J.Fluid Mech. 55, 385-400 (1972).

[5] Carrier, G.F., Krook, M., Pearson, C.E. Functions of a complex variable: Theory and technique. Hod Press: Icatha, New York (1983).

[6] Chivilikhin, S.A. Dynamics of a free multiply connected surface of a viscous liquid. Sov. Phys. Dokl. 35, 923-924 (1990).

[7] Crowdy, D.G. This volume (1999).

[8] Crowdy, D.G. \& Tanveer, S. A theory of exact solutions for plane viscous blobs. J. Nonlinear Sci. 8, 261-279 (1998).

[9] Cummings, L.J. Free boundary models in viscous flow. D. Phil. Thesis, University of Oxford (1996).

[10] Cummings, L.J., Howison, S.D., King, J.R. Conserved quantities in Stokes flow with free surfaces. Phys. Fluids 9, 477-480 (1997).

[11] Davis, P.J. The Schwarz function and its applications. Carus Math. Monographs 17, Math. Assoc. of America (1974).

[12] Duren, P.L. Univalent functions. Springer-Verlag (1983).

[13] Elliott, C.M. \& Janovsky, V. A variational inequality approach to Hele-Shaw flow with a moving boundary. Proc. Roy. Soc. Edin. A - Math. 88, 93-107 (1981).

[14] Elliott, C.M. \& OCKendon, J.R. Weak and variational methods for moving boundary problems. Pitman, London (1982).

[15] Entov, V.M., Etingof, P.I. \& Kleinbock, D.Ya. Hele-Shaw flows with a free boundary produced by multipoles. Europ. J. Appl. Math. 4, 97-120 (1993).

[16] Galin, L.A. Unsteady filtration with a free surface. Dokl. Akad. Nauk. S.S.S.R. 47, 246-249 (in Russian) (1945).

[17] Garabedian, P.R. Free boundary flows of a viscous liquid. Comm. Pure $E S$ Appl. Math. XIX, 421-434 (1966).

[18] Hohlov, Yu.E., Howison, S.D. On the classification of solutions to the zero surface tension model for Hele-Shaw free boundary flows. Quart. Appl. Math. 51, 777-789 (1993).

[19] Hohlov, Yu.E., Howison, S.D., Huntingford, C., Ockendon, J.R. \& Lacey, A.A. A model for non-smooth free boundaries in Hele-Shaw flow. Q. Jl. Mech. Appl. Math. 47, 107-128 (1994).

[20] Hopper, R.W. Coalescence of two equal cylinders: exact results for creeping flow driven by capillarity. J. Am. Ceram. Soc. 67, C262-264 (1985).

[21] Hopper, R.W. Plane Stokes flow driven by capillarity on a free surface. J. Fluid Mech. 213, 349-375 (1990).

[22] Howison, S.D., Ockendon, J.R. \& Lacey, A.A. Singularity development in moving boundary problems. Q. Jl. Mech. Appl. Math. 38, 343-360 (1985).

[23] Howison, S.D. Cusp development in Hele-Shaw flow with a free surface. SIAM J. Appl. Math. 46, 20-26 (1986). 
[24] Howison, S.D. Complex variable methods in Hele-Shaw moving boundary problems. Europ. J. Appl. Math. 3, 209-224 (1992).

[25] Howison, S.D. \& Richardson, S. Cusp development in free boundaries, and twodimensional slow viscous flows. Europ. J. Appl. Math. 6, 441-454 (1995).

[26] Howison, S.D., Morgan, J.D. \& Ockendon, J.R.. A class of codimension two free boundary problems, SIAM Review 39, 221-253, (1997).

[27] JeOng, J. \& Moffatt, H.K. Free-surface cusps associated with flow at low Reynolds number. J. Fluid Mech. 241, 1-22 (1992).

[28] KIng, J.R. Mathematical aspects of semiconductor process modelling, D. Phil. Thesis, University of Oxford (1986).

[29] King, J.R., Lacey, A.A. \& Vazquez, J.L. Persistence of corners in free boundaries in Hele-Shaw flow. Europ. J. Appl. Math. 6, 455-490 (1995).

[30] King, J.R. Development of singularities in some moving boundary problems. Europ. J. Appl. Math. 6, 491-507 (1995).

[31] LACEY, A.A. Moving boundary problems in the flow of liquid through porous media. $J$. Austral. Math. Soc. B24, 171-193 (1982).

[32] Langlois, W.E. Slow Viscous Flow. Macmillan (1964).

[33] Milne-Thomson, L.M. Theoretical Hydrodynamics (5th edition). Dover (1996).

[34] Paterson, L. Radial fingering in a Hele-Shaw cell. J. Fluid Mech. 113, 513-529 (1981).

[35] PitTs, E. Penetration of fluid into a Hele-Shaw cell: the Saffman-Taylor experiment. J.Fluid Mech. 97, 53-64 (1980).

[36] Polubarinova-Kochina, P.Ya. On the motion of the oil contour. Dokl. Akad. Nauk. S.S.S.R. 47, 254-257 (in Russian) (1945).

[37] Richardson, S. Two-dimensional bubbles in slow viscous flows. J. Fluid Mech. 33, 476-493 (1968).

[38] Richardson, S. Hele-Shaw flows with a free boundary produced by the injection of fluid into a narrow channel. J. Fluid Mech. 56, 609-618 (1972).

[39] Richardson, S. Two-dimensional bubbles in slow viscous flows. Part 2. J. Fluid Mech. 58, 115-127 (1973).

[40] Richardson, S. Some Hele-Shaw flows with time-dependent free boundaries. J. Fluid Mech. 102, 263-278 (1981).

[41] Richardson, S. Two-dimensional slow viscous flows with time-dependent free boundaries driven by surface tension. Europ. J. Appl. Math. 3, 193-207 (1992).

[42] Richardson, S. Hele-Shaw flows with time-dependent free boundaries involving injection through slits. Studies Appl. Math. 87, 175-194 (1992).

[43] Richardson, S. Hele-Shaw flows with time-dependent free boundaries in which the fluid occupies a multiply-connected region. Europ. J. Appl. Math. 5, 97-122 (1994).

[44] Richardson, S. Two-dimensional Stokes flows with time-dependent free boundaries driven by surface tension. Europ. J. Appl. Math. 8, 311-330 (1997).

[45] Richardson, S. On the classification of solutions to the zero-surface tension model for Hele-Shaw free boundary flows. Quart. Appl. Math. 55, 313-319 (1997).

[46] Richardson, S. Plane Stokes flow with time-dependent free boundaries in which the fluid occupies a doubly-connected domain. Preprint (1999).

[47] Schaeffer, D.G. Some examples of singularities in a free boundary. Ann. Scuol. Norm. Sup. Pisa 4, 133-144 (1977).

[48] Tanveer, S. \& Vasconcelos, G.L. Time-evolving bubbles in two dimensional Stokes flow. J. Fluid Mech. 301, 325-344 (1995).

[49] Youngren, G.K. \& Acrivos, A. On the shape of a gas bubble in a viscous extensional flow. J.Fluid Mech. 73, 433-442 (1976). 